Correspondence John H. Brumell john.brumell@sickkids.ca

Received 13 March 2006

Revised 3 May 2006

Accepted 4 May 2006

\section{Mutational analysis of Salmonella translocated effector members SifA and SopD2 reveals domains implicated in translocation, subcellular localization and function}

\author{
Nat F. Brown, ${ }^{1} \dagger$ Jason Szeto, ${ }^{2} \dagger$ Xiuju Jiang, ${ }^{2}$ Brian K. Coombes, ${ }^{3}$ \\ B. Brett Finlay ${ }^{1}$ and John $H$. Brumell ${ }^{2,4}$ \\ ${ }^{1}$ Michael Smith Laboratories and Departments of Biochemistry and Molecular Biology, \\ Microbiology, and Immunology, University of British Columbia, Vancouver, British Columbia V6T \\ 1Z4, Canada \\ ${ }^{2}$ Infection, Immunity, Injury, and Repair Program, The Hospital for Sick Children, 555 University \\ Avenue, Toronto, Ontario M5G 1X8, Canada \\ ${ }^{3}$ Department of Biochemistry and Biomedical Sciences, McMaster University, Hamilton, Ontario \\ L8N 3Z5, Canada \\ ${ }^{4}$ Department of Medical Genetics and Microbiology, University of Toronto, Toronto, Ontario \\ M5S 1A8, Canada
}

\begin{abstract}
Salmonella enterica serovar Typhimurium is a facultative intracellular pathogen causing disease in several hosts. These bacteria use two distinct type III secretion systems that inject effector proteins into the host cell for invasion and to alter maturation of the Salmonella-containing vacuole. Members of the Salmonella translocated effector (STE) family contain a conserved N-terminal translocation signal of approximately 140 aa. In this study, the STE family member SifA was examined using deletion strategies. Small deletions (approx. 20 residues long) throughout SifA were sufficient to block its secretion and/or translocation into host cells. Transfection of HeLa cells with a GFP-SifA fusion was previously shown to be sufficient to induce formation of Sif-like tubules resembling structures present in Salmonella-infected cells. The present study showed that both $\mathrm{N}$ - and $\mathrm{C}$-terminal domains of SifA are required for this phenotype. Furthermore, both domains could induce aggregation of Lamp1-positive compartments, provided they were coupled to the minimal C-terminal membrane-anchoring motif of SifA. Mutation or deletion of the conserved STE N-terminal WEK(I/M)xxFF translocation motif of SopD2 disrupted its association with Lamp1-positive compartments, implicating these residues in both effector translocation and subcellular localization. Interestingly, one GFP-SifA deletion mutant lacking residues 42-101, but retaining the WEK(I/M)xxFF motif, targeted the Golgi apparatus. In addition, short peptides containing the signature WEK(I/M)xxFF motif derived from the N-termini of Salmonella effectors SopD2, SseJ and SspH2 were sufficient to localize GFP to the Golgi. These studies suggest that Salmonella effectors contain multifunctional motifs or domains that regulate several effector traits, including protein secretion/translocation, localization and subversion of host cell systems. Conditions that perturb the tertiary structure of effectors can influence their localization in host cells by liberating cryptic intracellular targeting motifs.
\end{abstract}

\title{
INTRODUCTION
}

The facultative intracellular pathogen Salmonella enterica serovar Typhimurium (serovar Typhimurium) is respon-

†These authors contributed equally to the work.

Abbreviations: BFA, brefeldin A; Lamp1, lysosome-associated membrane protein 1; SCV, Salmonella-containing vacuole; Sif, Salmonellainduced filament; SPI, Salmonella pathogenicity island; STE, Salmonella translocated effector; T3SS, type III secretion system; TAPAS-1, tryptophan anchoring phosphatidic acid selective binding domain 1. sible for a range of diseases in different hosts, including gastroenteritis in humans and a typhoid-fever-like disease in mice (Tsolis et al., 1999). From mouse models, infection by serovar Typhimurium arises from bacteria crossing the intestinal epithelial barrier through M-cells or by luminal 
sampling of intestinal contents by $\mathrm{CD} 18^{+}$phagocytes (Jones et al., 1994; Vasquez-Torres et al., 1999). After breaching the epithelium, serovar Typhimurium goes on to colonize the liver and spleen of infected mice, residing within phagocytic cells at these locations (Richter-Dahlfors et al., 1997; Salcedo et al., 2001).

During the course of disease, serovar Typhimurium relies on two distinct type III secretion systems (T3SSs) to translocate bacterial-encoded virulence factors, termed effectors, directly from the bacterial cytosol into the host cell cytosol (Galan, 2001). The T3SS encoded by Salmonella pathogenicity island 1 (SPI-1) is required for invasion of epithelial cells, secreting several effectors that modulate host actin rearrangements and membrane ruffling to facilitate bacterial uptake (Bakshi et al., 2000; Friebel et al., 2001; Galan \& Fu, 2000; Galan \& Zhou, 2000; Hardt et al., 1998; Stender et al., 2000).

Upon entry into host cells, serovar Typhimurium resides in a membrane-bound compartment termed the Salmonellacontaining vacuole (SCV) (Dunlap et al., 1991; Knodler \& Steele-Mortimer, 2003; Takeuchi, 1967). Expression of a second T3SS, encoded on another pathogenicity island, SPI2 , is induced within the SCV and is essential for intracellular growth and virulence of serovar Typhimurium (Cirillo et al., 1998; Hensel et al., 1998; Ochman et al., 1996; Shea et al., 1996, 1999; Waterman \& Holden, 2003). The SPI-2 T3SS has been shown to be required for many events characteristic of Salmonella infection, including the modulation of host endocytic traffic (Brumell et al., 2001b, 2002; Brumell \& Grinstein, 2004; Garcia-del Portillo et al., 1993; Knodler \& Steele-Mortimer, 2003) and evasion of host cell reactive oxygen species and inducible nitric oxide synthase (Chakravortty et al., 2002; Gallois et al., 2001; VazquezTorres et al., 2000, 2001).

SPI2-T3SS effectors are translocated across the SCV membrane and into the host cell (Cirillo et al., 1998; Miao \& Miller, 2000; Waterman \& Holden, 2003). One such effector, SifA, is essential for inducing the extension of long membranous tubules called Salmonella-induced filaments (Sifs) that emanate from the SCV along microtubule networks (Brumell et al., 2002; Garcia-del Portillo et al., 1993; Stein et al., 1996). Transfection of SifA-GFP into host cells is sufficient to mimic the phenotype of the translocated effector, causing aggregation of late endocytic compartments and the formation of Sif-like tubules (Brumell et al., 2001a, 2002). Membrane binding by SifA is mediated by a C-terminal hexapeptide membrane anchor containing an $S$-acylation site and a CAAX motif serving as a target for host cell prenylation (Boucrot et al., 2003; Reinicke et al., 2005). Sifs are decorated with markers typically found on late endocytic compartments including Rab7 and Lamp1, suggesting that their formation arises in part from the fusion of the SCV with these compartments (Brumell et al., 2003; Brumell \& Grinstein, 2004; Garcia-del Portillo et al., 1993). Sifs are associated with rapidly replicating bacteria and their formation peaks at $8-10 \mathrm{~h}$ post-infection (Birmingham et al., 2005). Deletion of sifA significantly attenuates serovar Typhimurium virulence, impairs bacterial replication in macrophages, abrogates Sif formation and destabilizes the SCV, suggesting that the control of SCV membrane dynamics is crucial for pathogenicity (Beuzon et al., 2000, 2002; Brumell et al., 2001a; Stein et al., 1996). SifA has been shown to interact with the Rab7 GTPase and may promote the outward growth of Sifs by uncoupling Sif-associated Rab7 from Rab7-interacting lysosomal protein (RILP) (Harrison et al., 2004). Detachment from the centripetally directed dynein motor complex normally associated with RILP may allow Sif extension to proceed throughout the host cell (Harrison et al., 2004). SifA also interacts with a host protein termed 'SifA and kinesin-interacting protein' (SKIP). Recruitment of SKIP to the SCV negatively regulates the recruitment of the plus-end-directed kinesin to this compartment, thus facilitating the inward migration of the SCV towards perinuclear regions of the host cell (Boucrot et al., 2005).

Much less is known about the function of other SPI-2 effectors in comparison to SifA. SopD2 contributes to virulence in mice and acts cooperatively with SifA to promote Sif formation (Brumell et al., 2003; Jiang et al., 2004). Similar to SifA, SopD2 associates with Sifs and late endocytic compartments in infected cells, whether transfected or delivered by serovar Typhimurium itself (Brumell et al., 2003; Jiang et al., 2004). A GFP-fusion to the first 75 aa of SopD2 has been shown to target late endocytic vesicles (Brumell et al., 2003). The SPI-2 effector SseJ also localizes to SCVs and Sifs when ectopically expressed in infected cells. In contrast to SopD2, SseJ appears to inhibit Sif formation through its deacylase activity, which may modify Sif membrane composition (Birmingham et al., 2005; Ohlson et al., 2005). Other SPI-2 effectors, such as SspH2, target the host cytoskeletal system. SspH2 interacts with the actin-binding proteins filamin and profilin and may be involved in regulating the dynamics of actin assembly around the internalized SCV (Miao et al., 2003).

The mechanism by which effector proteins are recognized by T3SSs is not well understood. In general, it appears that effector proteins contain two signals, one that directs protein secretion out of bacteria and into the surrounding medium (secretion signal), and another that is required to further direct protein delivery specifically into host cells (translocation signal) (Ghosh, 2004; Sory et al., 1995). The secretion signal is contained within the $5^{\prime}$ region of the gene, in a region encoding approximately the first 20 aa of the secreted substrate, though it is unclear whether the signal acts at the level of mRNA or protein (Anderson \& Schneewind, 1997; Ghosh, 2004; Lloyd et al., 2001; Ramamurthi \& Schneewind, 2003). Translocation signals appear to reside in regions downstream of secretion signals as well (Miao \& Miller, 2000; Sory et al., 1995). In particular, members of the Salmonella translocated effector (STE) family, including the SPI-2 effectors SifA, SopD2, SspH2 and SseJ, have a conserved N-terminus of $\sim 140$ aa that 
mediates translocation into host cells (Brumell et al., 2000, 2003; Miao \& Miller, 2000). Significantly, a conserved WEK(I/M)xxFF motif within the N-terminus of STE family members appears to be essential for effector translocation (Miao \& Miller, 2000). Interestingly, a fusion of the first 150 aa of SopD2 to GFP retains the ability to localize to late endosomal/lysosomal compartments, indicating that the $\mathrm{N}$-terminus is bifunctional and can mediate translocation and subcellular targeting (Brumell et al., 2003). However, the amino acid residues implicated in directing any of the STE family effectors to their specific subcellular host targets are currently unknown.

Many studies of bacterial effectors have relied on transfection to introduce epitope-tagged versions of effectors into host cells. This may be required due to the unavailability of specific antibodies for immunofluorescence, or low protein copy numbers that preclude their detection. For the most part, the activity and localization of effectors introduced by transfection correlates with that observed for bacterially translocated effectors, as exemplified by SifA (Brumell et al., 2001a, 2002). However, this is not always the case, as the effector SseG targets endosomal membranes, Sifs, SCVs and microtubules when delivered by bacteria (Kuhle \& Hensel, 2002; Kuhle et al., 2004), yet localizes to the Golgi when transfected into the same cell type (Salcedo \& Holden, 2003). This suggests that the context of effector protein delivery, such as the presence of other effectors, the influence of host responses and the nature of protein fusions, may be important for determining bacterial effector protein localization.

In this study, we performed functional analysis of SifA using deletion strategies to identify regions involved in effector secretion, translocation, localization and modulation of host endocytic trafficking. Although SifA belongs to the STE family, we show that domains throughout the protein, and not only within the $\mathrm{N}$-terminus, are required for secretion and translocation of this effector. We also provide evidence that either $\mathrm{N}$ - or C-terminal domains can associate with and aggregate endocytic compartments provided the SifA C-terminal membrane targeting motif is present; however, neither domain is sufficient to generate Sif-like tubules by themselves. In addition, we have determined that residues found within the conserved WEK(I/M)xxFF motif of SopD2 are required for the effector to target late endocytic compartments. Interestingly, a cryptic Golgi-binding peptide containing the above motif is also present in a subset of STE effectors. Our studies demonstrate that STE effectors have domains that possess multiple functions. Furthermore, intracellular targeting motifs within bacterial effectors can be liberated by conditions that may alter protein folding, or by the context in which it is presented.

\section{METHODS}

Bacterial strains, plasmids and growth conditions. The Salmonella enterica serovar Typhimurium strains used in this work were the wild-type strain SL1344 (Hoiseth \& Stocker, 1981), and
$\Delta$ sifA (Stein et al., 1996) and $\Delta s s a R$ (Brumell et al., 2001a), isogenic mutants derived from SL1344. Escherichia coli strains DH5 $\alpha$ or DH10B were used for all molecular cloning experiments. All bacteria were cultured in Luria-Bertani (LB) medium supplemented with chloramphenicol $\left(50 \mu \mathrm{g} \mathrm{ml}^{-1}\right)$, kanamycin $\left(50 \mu \mathrm{g} \mathrm{ml}^{-1}\right)$ or streptomycin $\left(100 \mu \mathrm{g} \mathrm{ml}^{-1}\right)$ where appropriate.

Plasmids used in this study are described in Table 1 and oligonucleotide sequences are described in Table 2. The plasmid pGFP-SifA was constructed by amplifying sifA from SL1344 chromosomal DNA and digesting the PCR product with KpnI and SacI, followed by ligating it into similarly digested pEGFP-C1. Small, in-frame, internal deletions were constructed in the sifA alleles carried on psifA-2HA and pGFPSifA by an approach analogous to inverse PCR using the oligonucleotides as indicated in Table 1. Following amplification the PCR products were digested with SpeI and ligated in an intramolecular fashion to generate plasmids containing the desired deletion. Plasmid pGFPSifA $\Delta 1-8$ was constructed by ligating a $\sim 0.5 \mathrm{~kb} K p n \mathrm{I}-S p e \mathrm{I}$ fragment

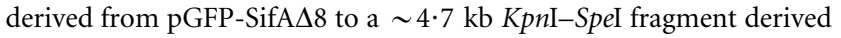
from pGFP-SifA $\Delta 1$. Plasmid pGFP-SifA $\Delta 1-5$ was constructed by ligating a $\sim 0.6 \mathrm{~kb} K p n \mathrm{I}-S p e \mathrm{I}$ fragment derived from pGFP-SifA $\Delta 5$ to a $\sim 4.7 \mathrm{~kb} \mathrm{Kp} n \mathrm{I}-S p e \mathrm{I}$ fragment derived from pGFP-SifA $\Delta 1$. Plasmid pGFP-SifA $\Delta 3-5$ was constructed by ligating a $\sim 0.6 \mathrm{~kb} K p n \mathrm{I}-S p e \mathrm{I}$ fragment derived from pGFP-SifA $\Delta 5$ to a $\sim 4 \cdot 8 \mathrm{~kb} K p n \mathrm{I}-S p e \mathrm{I}$ fragment derived from pGFP-SifA $\Delta 3$. Plasmid pGFP-SifA $\Delta 9-15$ was constructed by ligating a $\sim 0.5 \mathrm{~kb}$ SacI-SpeI fragment from pGFP-SifA $\Delta 9$ to a $\sim 4.7 \mathrm{~kb}$ SacI-Spel fragment derived from pGFP-SifA $\Delta 15$. Plasmid pGFP-SifA $\Delta 9-16$ was constructed by ligating a $\sim 0.5 \mathrm{~kb}$ SacI-SpeI fragment from pGFP-SifA $\Delta 9$ to a $\sim 4 \cdot 7 \mathrm{~kb}$ SacI-SpeI fragment derived from pGFP-SifA $\Delta 16$.

Deletions and mutations to sopD2 were generated using overlap extension strategies as outlined previously (Ho et al., 1989). Plasmid pSopD2(W37P,F44R)-GFP, encoding SopD2 bearing W37P and F44R substitutions, was created by amplifying overlapping sopD2 fragments from psopD2-GFP template in two separate PCRs using primer pairs listed in Tables 1 and 2. The overlapping segments were complementary to each other and contained the specific mutations. These fragments were subsequently pooled together and used as primers for another round of PCR with psopD2-GFP template to generate fulllength sopD2 bearing the desired mutations. After digestion with Bam HI and $\mathrm{XhoI}$, the mutant gene was ligated into similarly treated pEGFP-N1 to form plasmid pSopD2(W37P,F44R)-GFP. A similar strategy was used to create pSopD2( $\triangle \mathrm{aa} 37-44)-\mathrm{GFP}$, which encodes SopD2 with amino acids 37-44 deleted. However, for this deletion one primer from each primer pair was designed such that its $3^{\prime}$ end annealed to the template on one side of the deletion, while the $5^{\prime}$ end would anneal to the template DNA on the other side of the deletion (Ho et al., 1989).

Plasmids pSopD2(aa31-64)-GFP, pSopD2(aa31-46)-GFP, pSopD(aa31-64)-GFP, pSifA(aa25-58)-GFP, pSseJ(aa28-61)-GFP and pSspH2(aa28-61)-GFP encode peptide regions from SopD2, SopD, SifA, SseJ and SspH2, respectively, fused to the N-terminus of GFP. These were constructed using template DNA from psopD2-GFP, psopD-GFP, GFP-SifA (Table 1), or from wild-type serovar Typhimurium SL1344 chromosomal DNA as required. PCR amplicons were digested with BamHI and SalI and ligated into similarly digested pEGFP-N1.

Cell culture, infection of cultured cells and transfections. HeLa and RAW264.7 cell lines were obtained from the ATCC, and were cultured in Dulbecco's modified Eagle's medium (DMEM) supplemented with fetal calf serum at $10 \%(\mathrm{v} / \mathrm{v})$. Cell cultures were incubated at $37^{\circ} \mathrm{C}$ and $5 \% \mathrm{CO}_{2}$. Infections of HeLa cells were performed as described previously (Steele-Mortimer et al., 1999). HeLa cells were seeded at $5 \times 10^{4}$ cells per well in a 24 -well cell culture 
Table 1. Plasmids used in this study

\begin{tabular}{|c|c|c|}
\hline Plasmid & Description & Reference \\
\hline pEGFP-C1 & $g f p$, for constructing fusions to the $\mathrm{C}$ terminus of $\mathrm{GFP}, \mathrm{Km}^{\mathrm{R}}$ & Clontech \\
\hline pEGFP-N1 & $g f p$, for constructing fusions to the $\mathrm{N}$ terminus of $\mathrm{GFP}, \mathrm{Km}^{\mathrm{R}}$ & Clontech \\
\hline psifA-2HA & sifA(1-408)::2HA :: sifA(409-1011), pACYC derivative, $\mathrm{Cm}^{\mathrm{R}}$ & Brumell et al. (2002) \\
\hline pGFP-SifA & $\begin{array}{l}g f p:: \text { sifA, pEGFP-C1 derivative containing sifA amplified using oligonucleotides } \\
\text { sifA-f2 and sifA-r2, } \mathrm{Km}^{\mathrm{R}}\end{array}$ & This study \\
\hline psifA-2HA $\Delta 1$ & $\begin{array}{l}\text { sifA(1-408)::2HA:: sifA(409-1011) } \Delta 4-63(\text { relative to wt sifA), psifA-2HA derivative } \\
\text { using oligonucleotides sifam } 1 \mathrm{f} 2 \mathrm{~b} \text { and sifam } 1 \mathrm{r}, \mathrm{Cm}^{\mathrm{R}}\end{array}$ & This study \\
\hline psifA-2HA $\Delta 2$ & $\begin{array}{l}\text { sifA }(1-408):: 2 \mathrm{HA}: \text { : sifA }(409-1011) \Delta 64-123(\text { relative to wt sifA), psifA-2HA derivative } \\
\text { using oligonucleotides sifam } 2 \mathrm{f} \text { and } \operatorname{sifam} 2 \mathrm{r}, \mathrm{Cm}^{\mathrm{R}}\end{array}$ & This study \\
\hline psifA-2HA $\Delta 3$ & $\begin{array}{l}\text { sifA }(1-408):: 2 \mathrm{HA}:: \operatorname{sifA}(409-1011) \Delta 124-183(\text { relative to wt sifA), psifA-2HA derivative } \\
\text { using oligonucleotides sifam } 3 \mathrm{f} \text { and } \operatorname{sifam} 3 \mathrm{r}, \mathrm{Cm}^{\mathrm{R}}\end{array}$ & This study \\
\hline psifA-2HA $\Delta 4$ & $\begin{array}{l}\text { sifA }(1-408):: 2 \mathrm{HA}: \text { : sifA }(409-1011) \Delta 184-243(\text { relative to wt sifA), psifA-2HA derivative } \\
\text { using oligonucleotides sifam } 4 \mathrm{f} \text { and sifam } 4 \mathrm{r}, \mathrm{Cm}^{\mathrm{R}}\end{array}$ & This study \\
\hline psifA-2HA $\Delta 5$ & $\begin{array}{l}\text { sifA }(1-408):: 2 \mathrm{HA}:: \operatorname{sifA}(409-1011) \Delta 244-303(\text { relative to wt sifA), psifA-2HA derivative } \\
\text { using oligonucleotides sifam } 5 \mathrm{f} \text { and } s i f a m 5 \mathrm{r}, \mathrm{Cm}^{\mathrm{R}}\end{array}$ & This study \\
\hline psifA-2HA $\Delta 6$ & $\begin{array}{l}\text { sifA }(1-408):: 2 \mathrm{HA}: \text { : sifA }(409-1011) \Delta 304-363(\text { relative to wt sifA), psifA-2HA derivative } \\
\text { using oligonucleotides sifam6f and sifam6r, } \mathrm{Cm}^{\mathrm{R}}\end{array}$ & This study \\
\hline psifA-2HA $\Delta 7$ & $\begin{array}{l}\text { sifA }(1-408):: 2 \mathrm{HA}:: \operatorname{sifA}(409-1011) \Delta 433-492(\text { relative to wt sifA), psifA-2HA derivative } \\
\text { using oligonucleotides sifam7f and } s i f a m 7 \mathrm{r}, \mathrm{Cm}^{\mathrm{R}}\end{array}$ & This study \\
\hline psifA-2HA $\Delta 8$ & $\begin{array}{l}\text { sifA }(1-408):: 2 \mathrm{HA}: \text { : sifA }(409-1011) \Delta 493-552(\text { relative to wt sifA), psifA-2HA derivative } \\
\text { using oligonucleotides sifam } 8 \mathrm{f} \text { and } s i f a m 8 \mathrm{r}, \mathrm{Cm}^{\mathrm{R}}\end{array}$ & This study \\
\hline psifA-2HA $\Delta 9$ & $\begin{array}{l}\text { sifA }(1-408):: 2 \mathrm{HA}:: \operatorname{sifA}(409-1011) \Delta 553-612(\text { relative to wt sifA), psifA-2HA derivative } \\
\text { using oligonucleotides sifam9f and sifam9r, } \mathrm{Cm}^{\mathrm{R}}\end{array}$ & This study \\
\hline psifA-2HA $\Delta 10$ & $\begin{array}{l}\text { sifA }(1-408):: 2 \mathrm{HA}: \text { : sifA }(409-1011) \Delta 613-672(\text { relative to wt sifA), psifA-2HA derivative } \\
\text { using oligonucleotides sifam } 10 \mathrm{f} \text { and sifam } 10 \mathrm{r}, \mathrm{Cm}^{\mathrm{R}}\end{array}$ & This study \\
\hline psifA-2HA $\Delta 11$ & $\begin{array}{l}\text { sifA }(1-408):: 2 \mathrm{HA}: \text { : sifA }(409-1011) \Delta 673-732(\text { relative to wt sifA), psifA-2HA derivative } \\
\text { using oligonucleotides sifam } 11 \mathrm{f} \text { and sifam } 11 \mathrm{r}, \mathrm{Cm}^{\mathrm{R}}\end{array}$ & This study \\
\hline psifA-2HA $\Delta 12$ & $\begin{array}{l}\text { sifA }(1-408):: 2 \mathrm{HA}:: \operatorname{sifA}(409-1011) \Delta 733-792(\text { relative to wt sifA), psifA-2HA derivative } \\
\text { using oligonucleotides sifam } 12 \mathrm{f} \text { and } s i f a m 12 \mathrm{r}, \mathrm{Cm}^{\mathrm{R}}\end{array}$ & This study \\
\hline psifA-2HA $\Delta 13$ & $\begin{array}{l}\text { sifA(1-408)::2HA : :sifA(409-1011) } \Delta 793-852(\text { relative to wt sifA), psifA-2HA derivative } \\
\text { using oligonucleotides sifam } 13 \mathrm{f} \text { and sifam } 13 \mathrm{r}, \mathrm{Cm}^{\mathrm{R}}\end{array}$ & This study \\
\hline psifA-2HA $\Delta 14$ & $\begin{array}{l}\text { sifA }(1-408):: 2 \mathrm{HA}:: \operatorname{sifA}(409-1011) \Delta 853-912(\text { relative to wt sifA), psifA-2HA derivative } \\
\text { using oligonucleotides sifam } 14 \mathrm{f} \text { and sifam } 14 \mathrm{r}, \mathrm{Cm}^{\mathrm{R}}\end{array}$ & This study \\
\hline psifA-2HA $\Delta 15$ & $\begin{array}{l}\text { sifA }(1-408):: 2 \mathrm{HA}: \text { : sifA }(409-1011) \Delta 913-972(\text { relative to wt sifA), psifA-2HA derivative } \\
\text { using oligonucleotides sifam } 15 \mathrm{f} \text { and } \operatorname{sifam} 15 \mathrm{r}, \mathrm{Cm}^{\mathrm{R}}\end{array}$ & This study \\
\hline psifA-2HA $\Delta 16$ & $\begin{array}{l}\text { sifA }(1-408):: 2 \mathrm{HA}:: \operatorname{sifA}(409-1011) \Delta 973-1008 \text { (relative to wt sifA), psifA-2HA } \\
\text { derivative using oligonucleotides sifam } 16 \mathrm{f} \text { and sifam } 16 \mathrm{r} 2, \mathrm{Cm}^{\mathrm{R}}\end{array}$ & This study \\
\hline pGFP-SifA $\Delta 1$ & $\begin{array}{l}g f p:: \text { sifA } \triangle 4-63 \text { (relative to wt sifA), pGFP-SifA derivative using oligonucleotides } \\
\text { sifam } 1 \mathrm{f} 3 \text { and sifam } 1 \mathrm{r}, \mathrm{Km}^{\mathrm{R}}\end{array}$ & This study \\
\hline pGFP-SifA $\Delta 5$ & $\begin{array}{l}\text { gfp:: sifA } \Delta 244-303(\text { relative to wt sifA), pGFP :: SifA derivative using oligonucleotides } \\
\text { sifam5f and sifam } 5 \mathrm{r}, \mathrm{Km}^{\mathrm{R}}\end{array}$ & This study \\
\hline pGFP-SifA $\Delta 8$ & $\begin{array}{l}\text { gfp:: sifA } \Delta 493-552(\text { relative to wt sifA), pGFP :: SifA derivative using oligonucleotides } \\
\text { sifam8f and sifam } 8 \mathrm{r}, \mathrm{Km}^{\mathrm{R}}\end{array}$ & This study \\
\hline pGFP-SifA $\Delta 9$ & $\begin{array}{l}g f p: \text { : sifA } 553-612(\text { relative to wt sifA), pGFP-SifA derivative using oligonucleotides } \\
\text { sifam9f and sifam9r, } \mathrm{Km}^{\mathrm{R}}\end{array}$ & This study \\
\hline pGFP-SifA $\Delta 15$ & $\begin{array}{l}g f p: \text { : sifA } \Delta 913-972(\text { relative to wt sifA), pGFP : : SifA derivative using oligonucleotides } \\
\text { sifam15f and sifam15r, } \mathrm{Km}^{\mathrm{R}}\end{array}$ & This study \\
\hline pGFP-SifA $\Delta 16$ & $\begin{array}{l}g f p:: \text { sifA } \Delta 973-1008(\text { relative to wt sifA), pGFP : : SifA derivative using oligonucleotides } \\
\text { sifam16f and sifam16r1, } \mathrm{Km}^{\mathrm{R}}\end{array}$ & This study \\
\hline pGFP-SifA $\Delta 1--5$ & $g f p::$ sifA $\Delta 4-303$ (relative to wt sifA), pGFP-SifA $\Delta 1$ derivative, $\mathrm{Km}^{\mathrm{R}}$ & This study \\
\hline pGFP-SifA $\Delta 1-8$ & $g f p:$ sifA $\Delta 4-552$ (relative to wt sifA), pGFP-SifA $\Delta 1$ derivative, $\mathrm{Km}^{\mathrm{R}}$ & This study \\
\hline pGFP-SifA $\Delta 3-5$ & $g f p:$ : sifA $\Delta 124-303$ (relative to wt sifA), pGFP-SifA $\Delta 3$ derivative, $\mathrm{Km}^{\mathrm{R}}$ & This study \\
\hline pGFP-SifA $\Delta 9-15$ & gfp:: sifA $\Delta 913-972$ (relative to wt sifA), pGFP-SifA $\Delta 15$ derivative, $\mathrm{Km}^{\mathrm{R}}$ & This study \\
\hline pGFP-SifA $\Delta 9-16$ & $g f p::$ sifA $\Delta 553-1008$ (relative to wt sifA), pGFP-SifA $\Delta 16$ derivative, $\mathrm{Km}^{\mathrm{R}}$ & This study \\
\hline
\end{tabular}


Table 1. cont.

\begin{tabular}{|c|c|c|}
\hline Plasmid & Description & Reference \\
\hline psopD2-2HA & sopD2:: $2 \mathrm{HA}, \mathrm{pACYC}$ derivative, $\mathrm{Cm}^{\mathrm{R}}$ & Brumell et al. (2003) \\
\hline psopD2-GFP & sopD2:: $g f p$, pEGFP-N1 derivative, $\mathrm{Km}^{\mathrm{R}}$ & Brumell et al. (2003) \\
\hline pSopD2(aa31-64)-GFP & $\begin{array}{l}\text { sopD2(91-192)::gfp (relative to wt sopD2), pEGFP-N1 derivative using oligo- } \\
\text { nucleotides sopD2-JS1 and sopD2-JS2, } \mathrm{Km}^{\mathrm{R}}\end{array}$ & This study \\
\hline pSopD2(aa31-46)-GFP & $\begin{array}{l}\text { sopD2(91-138)::gfp (relative to wt sopD2), pEGFP-N1 derivative using oligo- } \\
\text { nucleotides sopD2-JS1 and sopD2-JS3, } \mathrm{Km}^{\mathrm{R}}\end{array}$ & This study \\
\hline pSopD2( $\Delta$ aa37-44)-GFP & $\begin{array}{l}\text { sopD2( } \Delta 108-132):: g f p \text { (relative to wt sopD2), pEGFP-N1 derivative using oligo- } \\
\text { nucleotide pairs JB0111, sopD2 } \Delta 37-44 \text { upstream and JB0112, sopD2 } \Delta 37- \\
\text { 44downstream, } \mathrm{Km}^{\mathrm{R}}\end{array}$ & This study \\
\hline pSopD2(W37P,F44R)-GFP & $\begin{array}{l}\text { sopD2:: } g f p \text { encoding W37P and F44R substitutions (relative to wt SopD2), } \\
\text { pEGFP-N1 derivative using oligonucleotide pairs JB0111, sopD2-W37PF44R } \\
\text { and JB0112, sopD2-W37PF44Rrev, } \mathrm{Km}^{\mathrm{R}}\end{array}$ & This study \\
\hline psopD-GFP & $s o p D:: g f p$, pEGFP-N1 derivative, $\mathrm{Km}^{\mathrm{R}}$ & Brumell et al. (2003) \\
\hline pSopD(aa31-64)-GFP & $\begin{array}{l}\operatorname{sop} D(91-192):: g f p \text { (relative to wt sopD), pEGFP-N1 derivative using oligo- } \\
\text { nucleotides JS-SopD-FW and JS-SopD-RV, Km }{ }^{\mathrm{R}}\end{array}$ & This study \\
\hline pSspH2(aa28-61)-GFP & $\begin{array}{l}\text { sspH2(82-183) }:: g f p \text { (relative to wt sspH2), pEGFP-N1 derivative using oligo- } \\
\text { nucleotides JS-SspH2-FW and JS-SspH2-RV, } \mathrm{Km}^{\mathrm{R}}\end{array}$ & This study \\
\hline pSseJ(aa28-61)-GFP & $\begin{array}{l}\text { sseJ(82-183)::gfp (relative to wt sseJ), pEGFP-N1 derivative using oligonucleotides } \\
\text { JS-SseJ-FW and JS-SseJ-RV, } \mathrm{Km}^{\mathrm{R}}\end{array}$ & This study \\
\hline pSifA(aa25-58)-GFP & $\begin{array}{l}\text { sifA(73-174)::gfp (relative to wt sifA), pEGFP-N1 derivative using oligonucleotides } \\
\text { JS-SifA-FW and JS-SifA-RV, } \mathrm{Km}^{\mathrm{R}}\end{array}$ & This study \\
\hline
\end{tabular}

plate containing coverslips $16-24 \mathrm{~h}$ before infection. The bacteria used for HeLa cell infections were exponential-phase Salmonella grown for $3 \mathrm{~h}$ in LB medium. Bacteria were pelleted at $10000 \mathrm{~g}$ for $2 \mathrm{~min}$ and resuspended in PBS. The inoculum was diluted and added to HeLa cells at a m.o.i. of $100: 1$ at $37^{\circ} \mathrm{C}$ for $10 \mathrm{~min}$. The cells were then washed extensively with PBS, and growth medium containing $100 \mu \mathrm{g}$ gentamicin $\mathrm{ml}^{-1}$ was added until $2 \mathrm{~h}$ postinfection, at which point the cells were washed and growth medium containing $10 \mu \mathrm{g}$ gentamicin $\mathrm{ml}^{-1}$ was added for the duration of the experiment.

RAW264.7 cells were infected in triplicate with opsonized, stationaryphase bacteria. Bacterial cultures were grown for $16 \mathrm{~h}$ at $37^{\circ} \mathrm{C}$ and then opsonized in $20 \%$ normal human serum in DMEM for $25 \mathrm{~min}$ at $37^{\circ} \mathrm{C}$. RAW264.7 cells were inoculated with approximately 50 bacteria per cell followed by centrifugation for $5 \mathrm{~min}$ at $600 \mathrm{~g}$. Infected cells were incubated for $20 \mathrm{~min}$ at $37^{\circ} \mathrm{C}, 5 \% \mathrm{CO}_{2}$, washed three times with PBS and then cultured for $90 \mathrm{~min}$ in DMEM containing $100 \mu \mathrm{g}$ gentamicin $\mathrm{ml}^{-1}$. Cells were then washed as above and incubated in DMEM containing $10 \mu \mathrm{g}$ gentamicin $\mathrm{ml}^{-1}$ for an additional $20 \mathrm{~h}$. At 2 and $20 \mathrm{~h}$ post-infection, infected cells were washed and then lysed in $0 \cdot 25 \mathrm{ml} 1 \%$ Triton X-100, 0.1\% SDS in PBS. Lysates were diluted and plated in replicate onto LB agar for enumeration of c.f.u.

For transfection, HeLa cells were seeded at $5 \times 10^{4}$ cells per well into 24 -well cell culture plates containing coverslips. After 16-24 h, between 0.5 and $1 \mu \mathrm{g}$ DNA was used to transfect HeLa cells with Fugene 6 transfection reagent (Roche) according to the manufacturer's instructions. Following transfection, cells were cultured for a further 16-20 h. To enumerate the aggregation of Lamp1 ${ }^{+}$compartments or formation of Sif-like tubules, HeLa cells transfected with plasmids encoding various GFP-SifA deletion derivatives were fixed and immunostained for Lamp1 (see below). The numbers of transfected cells that contained Lamp1 ${ }^{+}$aggregates or Sif-like tubules were determined for at least 100 cells, and each experiment was performed at least three times. The assessment of swollen/ aggregated Lamp1 compartments is based on a subjective increase in Lamp $^{+}$compartment size. The mean \pm SD for these experiments is presented.

Immunofluorescence staining and microscopy. Immunofluorescence staining was carried out as described previously (Coombes et al., 2003). Samples were fixed in $2.5 \%$ paraformaldehyde/PBS solution (pH 7.4) for $10 \mathrm{~min}$ at $37^{\circ} \mathrm{C}$. Samples were then washed twice with PBS prior to being blocked and permeabilized in $10 \%$ normal goat serum, $0 \cdot 2 \%$ saponin in PBS (SS-PBS) for 1-16 h. Primary and secondary antibodies were overlaid on coverslips in SS-PBS for $1 \mathrm{~h}$, followed by three washes with PBS. Coverslips were mounted onto glass slides using DakoCytomation fluorescent mounting medium. Confocal microscopy was performed using a Zeiss Axiovert microscope $(63 \times$ objective $)$ and Zeiss LSM software. Images were imported into Adobe Photoshop 7 and assembled in Adobe Illustrator CS for labelling. For live cell imaging, HeLa cells were seeded onto $2.5 \mathrm{~cm}$ coverslips at $2.0 \times 10^{5}$ cells per well in a 6-well culture plate and transfected with $1.5 \mu \mathrm{g}$ pSopD2(aa31-64)-GFP (Table 1) as described above. After approximately $16 \mathrm{~h}$, seeded coverslips were transferred to RPMI medium (supplemented with L-glutamine, HEPES, no bicarbonate; Wisent). Cells were incubated with $5 \mu \mathrm{g}$ brefeldin A (BFA) $\mathrm{ml}^{-1}$ for $70 \mathrm{~min}$, followed by washout of the drug and replacement with fresh RPMI. Cells were imaged at 3 min intervals during the course of BFA treatment and during the recovery period following drug removal using a Leica DMIRE2 fluorescent microscope equipped with Openlab 3.1.7 software (Improvision).

Antibodies and reagents. The anti-Lamp1 (H4A3) and antitubulin (E7) antibodies were obtained from the Developmental Studies Hybridoma Bank, developed under the auspices of the NICHD, National Institutes of Health, and maintained by the University of Iowa (Department of Biological Sciences, Ames, IA, USA). H4A3 was used at 1:50-1:100 for immunofluorescence. 
Table 2. Oligonucleotides used in this study

\begin{tabular}{|c|c|}
\hline Name & Sequence $\left(5^{\prime}-3^{\prime}\right)$ \\
\hline sifA-f2 & ATGGAGCTCTGCCGATTACTATAGGGAATGG \\
\hline sifA-r2 & ATGGGTACCTTATAAAAAACAACATAAACAGCCG \\
\hline sifam $1 \mathrm{f} 2 \mathrm{~b}$ & ATGACTAGTCATATTAATCTCACTTATACTGGAGT \\
\hline sifamlf3 & ATGACTAGTCAGAGCTCGAGATCTGAG \\
\hline sifam $1 \mathrm{r}$ & ATGACTAGTACGAAAGAAGCATGGTG \\
\hline $\operatorname{sifam} 2 \mathrm{f}$ & ATGACTAGTATTCCTTGGGGAGTTGGTAAGG \\
\hline $\operatorname{sifam} 2 \mathrm{r}$ & ATGACTAGTGGCAAAGCAAAAGCGGAC \\
\hline sifam $3 f$ & ATGACTAGTAGTAGAAAAAAAGAAGTCTTTAAT \\
\hline sifam3r & ATGACTAGTCGAGAGCGGCTTACAGA \\
\hline sifam4f & ATGACTAGTTGTGGGGGCGCGTTCGG \\
\hline sifam $4 \mathrm{r}$ & ATGACTAGTGATAGATTTCAGGTTCATAATCCTC \\
\hline sifam $5 f$ & ATGACTAGTTCTTTGCGATGCGCAGGCT \\
\hline sifam5r & ATGACTAGTGATCAAAACGAAGAGAACGAATTG \\
\hline sifam6f & ATGACTAGTCATGATGCGAAGAATAATGGTG \\
\hline sifam6r & ATGACTAGTGAAGTCATGGGGAATCTTTATTT \\
\hline sifam7f & ATGACTAGTCATTTGTGGATGCGATTTT \\
\hline sifam7r & ATGACTAGTTGTCTCAATCCTGCTGGC \\
\hline sifam8f & ATGACTAGTTAATGTCGAAGGGAGGGG \\
\hline sifam8r & ATGACTAGTTTATATACTGAATTGAGAAAAGGAC \\
\hline sifam9f & ATGACTAGTATACCCCTCTATGTTGTCTAATGG \\
\hline sifam9r & ATGACTAGTTACCTGGCAGCGAAAATTCAG \\
\hline sifam10f & ATCACTAGTGGTTGCCTTTTCTTGCG \\
\hline sifam10r & ATGACTAGTAATATATCCGAAAGTACTCAGCAA \\
\hline sifaml1f & ATCACTAGTCGCATGGTGTAAAATGC \\
\hline sifam11r & ATGACTAGTAAACAGCTTGAAATACCACCAC \\
\hline sifam12f & ATCACTAGTTAATCCACACATCGCCATT \\
\hline sifam12r & ATGACTAGTGTTTTACTAGCGGATAAGACG \\
\hline sifam13f & ATGACTAGTCTCTTTTACCATTTTTTCAATAGG \\
\hline sifam13r & ATGACTAGTAGTATGTTAGCTGAGATAGTCGAAG \\
\hline sifam14f & ATGACTAGTTTGGCTGGTGCTGGGAT \\
\hline sifam14r & ATGACTAGTATAGACCCCCAGGCTATACAA \\
\hline sifam $15 f$ & ATGACTAGTTCTAAAAATGGCGTGAAAAACC \\
\hline sifam15r & ATGACTAGTTCAGAACAACAAAGCGGCTG \\
\hline sifam16f & ATGACTAGTGCGAACGTGTAGCGTGGTT \\
\hline sifam16r1 & ATGACTAGTTAAGGATCCACCGGATCTAGATAA \\
\hline sifam16r2 & ATGACTAGTTAAGTCGACCGATGCCCTT \\
\hline sopD2-JS1 & CGCGTCGACAGCATGGCGCTATACATGGGGGTATG \\
\hline sopD2-JS2 & CGCGGATCCGCTTCACATCCATGGATGAG \\
\hline sopD2-JS3 & CGCGGATCCGCTGTTCTGAAGCAGTCCTTAAATC \\
\hline JB0111 & CCGCTCGAGAGCATGCCAGTTACGTTAAGTTTTGGTAAT \\
\hline JB0112 & CGTGGATCCAGTATAAGCATATTGCGACAACTCGACTT \\
\hline sopD2 $\Delta$ aa37-44upstream & GTTTTTTGTGTGTTCTTACCCCCATGTATAGCGC \\
\hline sopD2 $\Delta$ aa37-44downstream & CTATACATGGGGGTAAGAACACACAAAAAACAAG \\
\hline sopD2-W37PF44R & GGGTACCGGATAGATTTAAGGACTGCCGCAGAACAC \\
\hline sopD2-W37PF44Rrev & GTGTTCTGCGGCAGTCCTTAAATCTATCCGGTACCC \\
\hline JS-SopD-FW & CGCGTCGACAGCATGGCAATCCATATGGGGGGTTGGG \\
\hline JS-SopD-RV & CGCGGATCCGCGCCCTGCCCGTGAATGATGGAGTG \\
\hline JS-SseJ-FW & CGCGTCGACAGCATGCTTCCGGAATTAAGTTTATGGG \\
\hline JS-SseJ-RV & CGCGGATCCGCTTCCTGATGGTGGTAAAGATTAAAGATACATTCG \\
\hline JS-SifA-FW & CGCGTCGACAGCATGGCATGGAAAGTTTTATGGG \\
\hline JS-SifA-RV & CGCGGATCCGCGCGTTCGGCAAACAACATCTCATG \\
\hline JS-SspH2-FW & CGCGTCGACAGCATGCCCCCTGAAATGAGTTCCTGGG \\
\hline JS-SspH2-RV & CGCGGATCCGCGGCCGGCGGGTGACAAATCGTCC \\
\hline
\end{tabular}


Rabbit anti-Lampl antibody (Affinity BioReagents) was used at $1: 200$ for immunofluorescence. Anti-tubulin antibody was used at a dilution of 1:5000 for Western blots. The anti-giantin antibody was used at 1:1000 for immunofluorescence and was obtained from Dr H. P. Hauri, University of Basel, Switzerland. The anti-HA epitope antibody (Covance) was used at 1:400 for immunofluorescence and $1: 2000$ for Western blots. Antibodies against DnaK and calnexin were obtained from Stressgen and used at 1:3500 and 1:2000, respectively, for Western blots. Alexa-568-conjugated goat antimouse and anti-rabbit antibodies were used at 1:200 and were purchased from Molecular Probes. Horseradish-peroxidase-labelled anti-mouse antibodies (Jackson Immunoresearch Laboratories) were used at a concentration of $1: 5000$.

In vitro secretion assays. In vitro secretion assays were carried out as described previously (Coombes et al., 2004). Bacteria were cultured until stationary phase in a modified M9 minimal medium optimized for SPI2 gene expression and SPI2 type III secretion (Beuzon et al., 1999; Nikolaus et al., 2001). Overnight cultures of the required Salmonella strains grown in LB broth were washed twice in low-phosphate, low-magnesium medium (LPM) and then inoculated $1: 50$ in $3 \mathrm{ml} \mathrm{LPM}$ at $\mathrm{pH} 5 \cdot 8$. LPM medium consisted of $5 \mathrm{mM} \mathrm{KCl}, 7 \cdot 5 \mathrm{mM}\left(\mathrm{NH}_{4}\right)_{2} \mathrm{SO}_{4}, 0.5 \mathrm{mM} \mathrm{K}_{2} \mathrm{SO}_{4}, 38 \mathrm{mM}$ glycerol $(0.3 \% \mathrm{v} / \mathrm{v}), 0 \cdot 1 \%$ Casamino acids, $8 \mu \mathrm{M} \mathrm{MgCl}_{2}, 337 \mu \mathrm{M} \mathrm{PO}_{4}^{3-}$, and $80 \mathrm{mM}$ MES (for titration to $\mathrm{pH} 5 \cdot 8$ ). Cultures were grown for $4-6 \mathrm{~h}$ at $37^{\circ} \mathrm{C}$ with shaking, after which the $\mathrm{OD}_{600}$ was measured. Bacteria were pelleted by centrifugation for $2 \mathrm{~min}$ at 12000 r.p.m. $\left(4{ }^{\circ} \mathrm{C}\right)$ and the supernatant was passed through a $0.22 \mu \mathrm{m}$ filter and precipitated with trichloroacetic acid $(10 \%, \mathrm{v} / \mathrm{v}$, final concentration) at $4{ }^{\circ} \mathrm{C}$ for $4-16 \mathrm{~h}$.

The trichloroacetic-acid-insoluble fraction was collected by centrifugation, washed with ice-cold acetone, and solubilized with a volume of $2 \times$ SDS-sample buffer ( $100 \mathrm{mM}$ Tris/ $\mathrm{HCl}, \mathrm{pH} 6 \cdot 8,20 \%$ glycerol, $4 \%$ SDS, $0.002 \%$ bromphenol blue and $200 \mathrm{mM}$ dithiothreitol) adjusted according to the $\mathrm{OD}_{600}$ of the original culture. When necessary, solubilized secreted proteins were neutralized with an appropriate volume of non-titrated Tris. The bacterial pellet fraction from above was also dissolved in a volume of $2 \times$ SDS-sample buffer adjusted according to the $\mathrm{OD}_{600}$ of the original culture. Proteins from equivalent numbers of bacterial cells, as determined by $\mathrm{OD}_{600}$ readings, were separated on $10 \%$ or $12 \%$ SDS-polyacrylamide gels, transferred to nitrocellulose membranes, and then blocked in Tris-buffered saline containing $0 \cdot 1 \%(\mathrm{v} / \mathrm{v})$ Tween $20($ TBST) and $5 \%(\mathrm{w} / \mathrm{v})$ powdered non-fat milk for $1 \mathrm{~h}$ at room temperature. Blots were incubated with mouse anti-HA monoclonal or mouse anti-DnaK monoclonal antibody in TBST plus $5 \%$ non-fat milk. Secondary antibodies conjugated to horseradish peroxidase were used at a 1:5000 dilution in TBST for $1 \mathrm{~h}$ at room temperature. Antibody complexes were detected using enhanced chemiluminescence (Amersham Biosciences).

Host cell fractionation. HeLa cells for fractionation were seeded at $2 \times 10^{6}$ per dish in $100 \mathrm{~mm}$ dishes, and were infected as described above. Cells were fractionated as previously described (Gauthier et al., 2000). In brief, cells were scraped and disrupted mechanically by passage through a 22-gauge needle in a homogenization buffer containing $250 \mathrm{mM}$ sucrose, $3 \mathrm{mM}$ imidazole and $0.5 \mathrm{mM}$ EDTA $(\mathrm{pH} 7 \cdot 4)$. The cell suspension was centrifuged at low speed $(3000 \mathrm{~g}$ for $15 \mathrm{~min}$ at $4{ }^{\circ} \mathrm{C}$ ) to pellet bacteria and unbroken cells (fraction designated ' $\mathrm{P}$ ' in these studies), followed by ultracentrifugation (41000 $\mathrm{g}$ for $20 \mathrm{~min}$ at $4{ }^{\circ} \mathrm{C}$ ) to separate cellular membranes (designated ' $\mathrm{M}$ ') from the cytosolic fraction (designated ' $\mathrm{C}$ '). Each fraction was dissolved in $1 \times$ SDS sample buffer and loaded onto $10 \%$ polyacrylamide gels for electrophoresis. Gels were transferred to PVDF membranes for Western blotting experiments.

\section{RESULTS}

\section{Deletions within SifA disrupt its secretion and translocation}

In general, the $\mathrm{N}$-termini of various bacterial T3SS effectors have been implicated in effector secretion and translocation (Cornelis \& Van Gijsegem, 2000; Miao \& Miller, 2000), while the actual functional effector domain appears to reside in the C-terminal half of the protein (Hueck, 1998). SifA has been classified as a member of the STE family, as it contains a conserved $\mathrm{N}$-terminal domain of approximately 140 aa that has been shown to be involved in translocation of another STE protein, SspH1 (Fig. 1a) (Miao \& Miller, 2000). The C-terminus of SifA bears similarity to several Gram-negative bacterial proteins, and contains a prenylation motif required for membrane insertion (Fig. 1a) (Boucrot et al., 2003; Brumell et al., 2002; Reinicke et al., 2005). Addition of an internal, double haemagglutinin tag $(2 \times \mathrm{HA}$; herein referred to as $2 \mathrm{HA})$ between the conserved $\mathrm{N}$-terminal type III secretion signal and the C-terminal portion of SifA resulted in a protein (SifA-2HA) that retained all known functions, including secretion, translocation, localization to endocytic compartments and induction of Sif formation (Brumell et al., 2002; Jiang et al., 2004). This suggested that SifA might follow the generalized model of T3SS effectors, and comprise at least two distinct domains, one implicated in its delivery into host cells, and the other in modulating host cell targets.

To gain more insight into functional domains of SifA, a panel of deletion mutants was constructed. A series of 16 genes encoding scanning internal deletions of SifA were constructed in psifA-2HA (Fig. 1b). Plasmids encoding these deletion mutants were transformed into a $\Delta$ sifA strain of serovar Typhimurium to determine their effects on the defective replication of this strain previously described in the RAW264.7 macrophage cell line (Brumell et al., 2001a). It was found that all deletions constructed in psifA-2HA resulted in reduced replication compared to the control strain containing the unmodified parental plasmid, which displayed expected replication levels within host cells (Fig. 2a). The psifA-2HA deletion plasmids were also unable to complement Sif formation and SCV maintenance in a chromosomal $\Delta$ siff strain when used to infect HeLa cells for $10 \mathrm{~h}$ (results not shown). Similarly, none of the sifA-2HA deletion alleles acted as dominant negatives on Sif formation and SCV maintenance when expressed from wild-type serovar Typhimurium used to infect HeLa cells (results not shown).

The inability to restore the intracellular growth of the $\Delta$ sifA strain to levels comparable to the $\Delta$ sifA strain containing unmodified psifA-2HA may be due to defects in secretion or translocation of each SifA-2HA mutant. Using an in vitro secretion assay optimized for SPI-2 gene expression and SPI2 T3SS secretion, we observed that the majority of deletions to SifA abrogated its secretion [Fig. 2b; compare bacterial pellet fractions $(\mathrm{P})$ with corresponding supernatants $(\mathrm{S})$ 
(a)

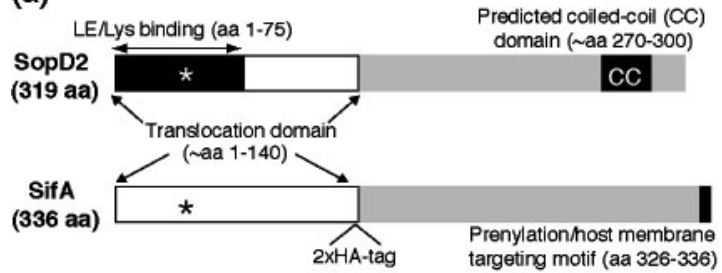

(b)

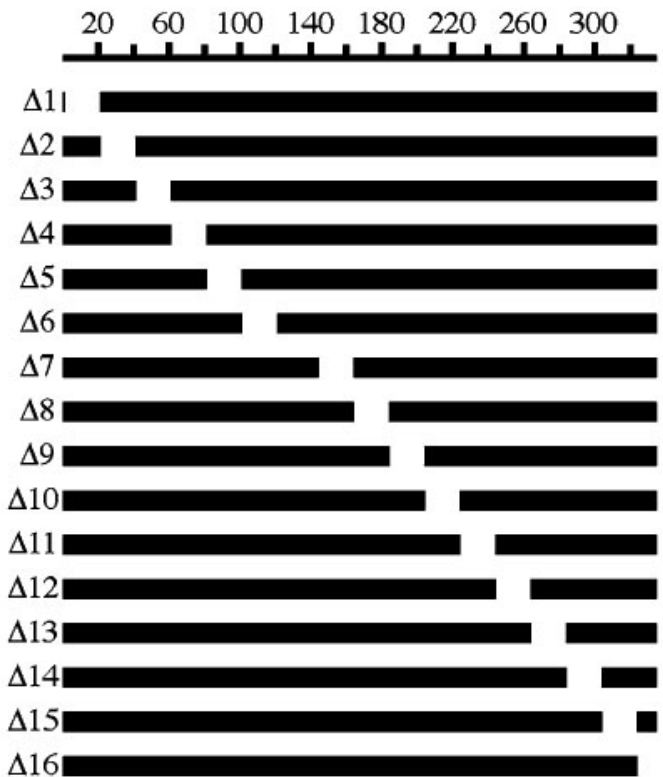

Fig. 1. Schematic of serovar Typhimurium effectors SifA and SopD2. (a) SifA and SopD2 share a conserved N-terminal region involved in translocation into host cells (Brumell et al., 2000; Miao \& Miller, 2000). SifA also contains a prenylation motif involved in host membrane targeting at its C-terminus (Boucrot et al., 2003; Reinicke et al., 2005). The position of an in-frame insertion of tandem HA-tags $(2 \times \mathrm{HA}$-tag) encoded in plasmid psifA-2HA is indicated in SifA (Brumell et al., 2002). The late-endosome/lysosome (LE/Lys)-binding region of SopD2, and a predicted C-terminal coiled-coil (CC) region are also shown. Asterisks indicate the relative position of a conserved WEK(I/M)xxFF motif found in members of the STE family. (b) Scale diagram indicating deletions constructed in each mutant SifA used in this study. The top line indicates scale in amino acids and represents wild-type SifA. Lines below represent SifA mutants, with the deleted region (20 aa in each case) indicated as a gap in the thick line. Mutant designations are labelled to the left and were constructed in both psifA-2HA and pGFP-SifA. Note that no deletion was constructed in the region of amino acids 122-144 due to the presence of the $2 \times$ HA epitope tag in SifA-2HA.

containing secreted proteins]. Western blotting using the anti-HA antibody showed that each SifA-2HA mutant was synthesized in the bacteria (Fig. 2b), confirming that our secretion results were not due to a lack of mutant protein being expressed. We note that limited amounts of mutant SifA-2HA were secreted from bacteria transformed with psifA-2HA $\Delta 11$, psifA-2HA $\Delta 15$ and psifA-2HA $\Delta 16$ compared to control bacteria carrying psifA-2HA (Fig. 2b). Interestingly, the mutant protein SifA- $2 \mathrm{HA} \Delta 2$, bearing a deletion of its conserved WEK $(\mathrm{I} / \mathrm{M}) \mathrm{xxFF}$ translocation domain common to STE effectors (Miao \& Miller, 2000), was also secreted by bacteria into the culture supernatant.

To detect the delivery of effector protein into host cells, we performed sensitive immunoblot-based translocation assays in HeLa cells using both wild-type serovar Typhimurium SL 1344 and a $\Delta s s a R$ strain deficient in SPI-2 type III secretion. Fig. 2(c) shows a representative assay testing the translocation of a SifA-2HA deletion derivative, namely the SifA$2 \mathrm{HA} \Delta 2$ mutant. Whereas wild-type SifA-2HA was delivered by the wild-type strain and could be detected in the host cell membrane fraction (M) by immunoblotting, SifA-2HA $\Delta 2$ was not. As expected, neither SifA-2HA nor SifA-2HA $\Delta 2$ was translocated from the $\Delta s s a R$ strain (SPI-2 T3SS defective) (Fig. 2c). The two signals that appear upon detection of wild-type SifA-2HA in lane 1 (Fig. 2c) likely represent post-translationally modified and unmodified forms of SifA-2HA. It is established that SifA undergoes prenylation and S-acylation within the host (Reinicke et al., 2005). Hence, the untranslocated SifA-2HA remaining within bacteria due to the SifA-2HA $\Delta 2$ mutation or the lack of a functional SPI-2 T3SS $(\Delta s s a R)$ was detected as the sole lower molecular mass signals common to lanes 4, 7 and 10 (Fig. 2c). The remaining SifA-2HA mutants were also tested using this immunoblot-based translocation assay, and were not translocated. This included mutants with deletions at the C-terminus outside of the conserved STE family protein translocation region (data not shown). Furthermore, immunofluorescence microscopy revealed that none of the SifA-2HA deletion proteins were translocated into host HeLa cells at 10 or $18 \mathrm{~h}$ postinfection when expressed from either a $\Delta$ sifA or a wild-type strain (data not shown). Collectively, these results show that regions throughout $\mathrm{SifA}$, and not only within its $\mathrm{N}$-terminus, are required for its secretion and/or translocation.

\section{$\mathrm{N}$ - and C-terminal domains of SifA each have roles in targeting and/or aggregation of Lamp1 ${ }^{+}$ compartments, but cannot induce Sif-like tubule formation by themselves}

Transfection of SifA-GFP results in its association with $\mathrm{Lamp}^{+}$vesicles that induces their aggregation and filamentation into Sif-like tubules, similar to bacterial delivery of the effector (Boucrot et al., 2003; Brumell et al., 2001a, 2002). A previous study has identified an 11 aa membraneanchoring motif at the C-terminus of SifA (Boucrot et al., 2003). Fusion of this motif to a cytosolic protein, GFP-SifB, was sufficient to target the protein to membrane compartments when transfected into HeLa cells (Boucrot et al., 2003). However, it is not clear whether these compartments were Lamp $1^{+}$and thus indicative of the actual subcellular targets of SifA.

To determine the domains in SifA involved in targeting and aggregating Lamp1 ${ }^{+}$vesicles, as well as in inducing Sif-like 

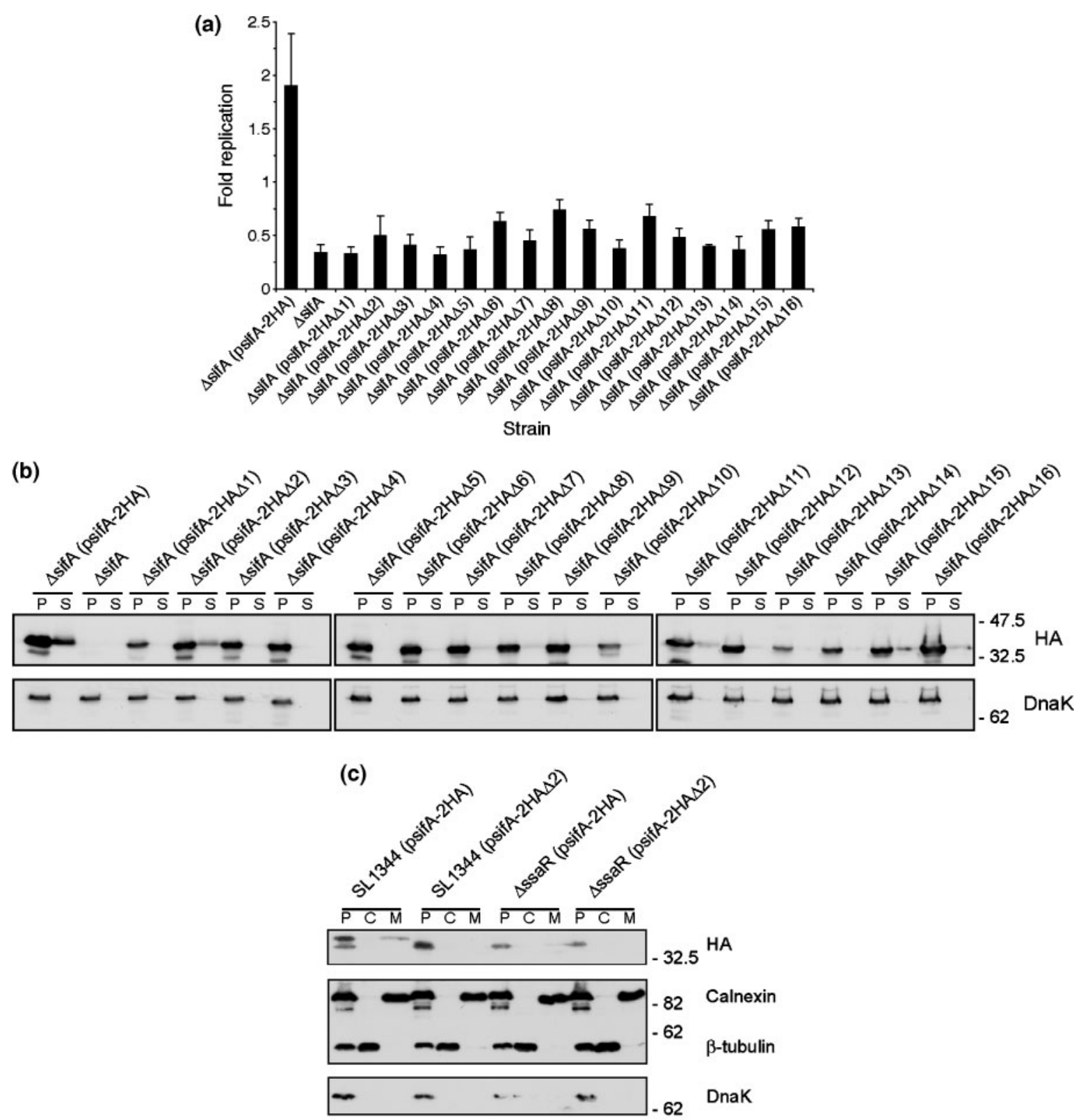

Fig. 2. Short deletions in SifA block its secretion and translocation into host cells. (a) Complementation of a chromosomal $\Delta$ sifA genotype with psifA-2HA deletion constructs. Replication of bacteria in RAW264.7 macrophages is expressed as the number of intracellular bacteria at $20 \mathrm{~h}$ divided by the number at $2 \mathrm{~h}$. The infecting strain is indicated below the graph, with the transformed plasmid indicated in parentheses. Results shown are means \pm SEM $(n=3)$. (b) Secretion of mutant SifA-2HA proteins into culture supernatants. The indicated strains were cultured until stationary phase in a minimal medium optimized for SPI-2 gene expression and SPI2 type III secretion. Bacteria were then centrifuged out of suspension and lysed in SDS sample buffer, and the culture supernatant proteins were precipitated using trichloroacetic acid and dissolved in SDS sample buffer. Samples were then resolved on $12 \%$ polyacrylamide gels, Western blotted and probed with anti-HA and anti-DnaK antibodies. Lanes are labelled ' $\mathrm{P}$ ' for whole cell bacterial samples and ' $\mathrm{S}$ ' for precipitated culture supernatant sample. DnaK was used as a control for cytosolic contamination of the culture supernatants. (c) Assessment of SifA-2HA $\Delta 2$ translocation into host cells. HeLa cells were infected for $18 \mathrm{~h}$ with SL1344 wild-type or $\Delta s s a R$ (SPI-2 T3SS-deficient mutant) transformed with psifA-2HA or psifA-2HA $\Delta 2$, as indicated. These were then fractionated into a low-speed pellet fraction designated ' $\mathrm{P}$ ', a high-speed pellet designated ' $\mathrm{M}$ ' and a supernatant fraction designated ' $\mathrm{C}$ ' as described in Methods. Fraction $\mathrm{P}$ primarily consists of high-order polymers, nuclei, unbroken cells and bacteria, fraction $\mathrm{M}$ consists mainly of host cell membranes and fraction C consists of soluble host cell cytoplasmic molecules. DnaK was used as a marker for potential bacterial contamination of fractions $\mathrm{M}$ and $\mathrm{C}$. Calnexin is an endoplasmic reticulum integral membrane protein and $\beta$-tubulin is a cytoplasmic protein. 
tubule formation, we constructed a GFP-fusion to the N-terminus of SifA (GFP-SifA). Several deletions to SifA were also constructed for localization studies in HeLa cells. Specifically, we tested the fusions GFP-SifA $\Delta$ 9-15 (lacking aa 185-324 of wild-type SifA), GFP-SifA $\Delta 9-16$ (lacking the C-terminus of SifA from aa 185), GFP-SifA $\Delta 1-8$ (lacking aa 2-184), GFP-SifA $\Delta 1-5$ (lacking aa 2-101), and GFPSifA $\Delta 3-5$ (lacking aa $42-101$ ) (Fig. 3a). GFP-SifA $\Delta 9-15$ was designed to retain the $\mathrm{C}$-terminal amino acids involved in membrane anchoring (Boucrot et al., 2003). HeLa transfectants were immunostained for Lamp1 to visualize colocalization and/or aggregation effects of each GFP-SifA fusion on late endocytic compartments.

As expected, wild-type GFP-SifA was associated with Lamp $1^{+}$vesicles, many of which appeared swollen and aggregated, as revealed by confocal microscopy and quantification (Fig. 3b, c). In addition, the fusion protein induced the formation of Sif-like tubules (Fig. 3b, d). These phenotypes elicited by GFP-SifA were consistent with those observed previously using a similar construct (Boucrot et al., 2003), and with SifA-GFP (Brumell et al., 2001a), where GFP was fused to the C-terminus of the effector. Hence, the functionality of both SifA fusions was similar (Fig. 3c). GFP-SifA $\Delta 9-15$ also produced a similar phenotype to the wild-type control (Fig. 3b, c), indicating that much of the C-terminus of SifA (from aa 185 to aa 324) is not required for binding and aggregating Lamp $1^{+}$compartments. However, GFP-SifA $\Delta 9-15$ could not induce the formation of Sif-like tubules. Removal of the SifA membrane-targeting motif from GFP-SifA $\Delta 9-15$, resulting in GFP-SifA $\triangle 9-16$ (Fig. 3a), rendered the protein distributed throughout the cytosol (Fig. 3b). Furthermore, GFPSifA $\triangle 9-16$ did not induce aggregation of Lamp $1^{+}$vesicle activities, similar to GFP transfectant controls (Fig. 3b, c). These results show that the $\mathrm{N}$-terminal half of SifA is able to aggregate Lamp $1^{+}$compartments provided the minimal SifA membrane-anchoring motif is present.

We next examined the effects of $\mathrm{N}$-terminal deletions to SifA using GPF-SifA constructs. Both microscopy and quantification showed that GFP-SifA $\Delta 1-8$ efficiently produced

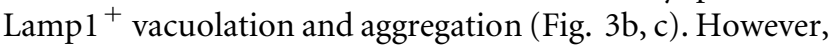
despite this ability, GFP-SifA $\Delta 1-8$ was not localized exclusively to Lamp $1^{+}$vesicles, and appeared in the cytosol as well (Fig. 3 b). GFP-SifA $\Delta 1-5$, which contained additional SifA N-terminal residues (aa 102-184) that were excluded from GFP-SifA $\Delta 1-8$ (Fig. 3a), also presented a cytosolic distribution with little colocalization with Lamp $1^{+}$vesicles (Fig. 3b). Curiously, GFP-SifA $\Delta 1-5$ did not induce significant aggregation of Lamp ${ }^{+}$compartments as GFP-SifA $\Delta 1-8$ did, in comparison to the negative control GFP (Fig. 3c). Significantly, these results indicate that the C-terminal half of SifA, which includes the membrane-targeting motif, is not sufficient to properly target and/or aggregate late endocytic compartments, and that determinants within the $\mathrm{N}$-terminus are required as well.
Interestingly, GFP-SifA $\Delta 3-5$ appeared concentrated at a region adjacent to the nucleus and did not colocalize with Lamp $1^{+}$compartments (Fig. 4). HeLa cells expressing GFPSifA $\Delta 3-5$ had significantly fewer Lamp $1^{+}$aggregates, even compared to cells transfected with the unmodified GFP vector (Fig. 3c). In contrast to both wild-type SifA-GFP and GFP-SifA, none of the GFP-SifA deletions could induce the formation of Sif-like tubules upon transfection of HeLa cells (Fig. 3d). Overall, our results suggest that both the N- and C-terminal domains of SifA contribute to its localization to, and aggregation of, Lamp $1^{+}$compartments. Furthermore, tubulation of Lamp1 ${ }^{+}$compartments into Sif-like tubules appears to require a complete SifA protein.

\section{GFP-SifA $\Delta 3-5$ targets the Golgi complex}

The localization of GFP-SifA $\Delta 3-5$ was strikingly different from that of the other GFP-SifA constructs. The fusion protein did not colocalize with Lamp1 ${ }^{+}$compartments, and was found predominantly at a juxtanuclear position and on vesicles surrounding the nucleus (Fig. 4, top panels). The concentration of GFP-SifA $\Delta 3-5$ adjacent to the nucleus suggested the fusion might be preferentially targeted to the Golgi apparatus. Indeed, the majority of GFP-SifA $\Delta 3-5$ colocalized with giantin (Fig. 4, bottom panels), an established marker of the Golgi (Linstedt \& Hauri, 1993; Seelig et al., 1994). In contrast to GFP-SifA $\Delta 1-5$, which was predominantly in the cytosol (Fig. 3b), the Golgi-targeting GFP-SifA $\Delta 3-5$ contained the first 41 aa of SifA (Fig. 3a). This suggested that determinants within the first 41 residues of SifA might redirect its subcellular localization in certain conditions, such as in the context of deletion mutations.

\section{A conserved $\mathbf{N}$-terminal motif in SopD2 is involved in membrane association and is sufficient to target the Golgi}

Similar to SifA, the SPI-2 effector SopD2 localizes to $\mathrm{Lamp1}^{+}$endocytic compartments when transfected as a GFP-fusion, or when delivered by the serovar Typhimurium T3SS (Brumell et al., 2003). We verified that such localization could occur simultaneously in HeLa cells by first transfecting SopD2-GFP and then infecting the cells with a $\triangle$ sopD2 serovar Typhimurium mutant delivering plasmidencoded SopD2-2HA. Both SopD2-GFP and SopD2-2HA were colocalized with Lamp1 ${ }^{+}$vesicles (Fig. 5b, middle row panels). Thus, SopD2 fusions to either epitope tag did not disrupt its targeting of late endocytic compartments.

Since the N-terminus of SifA contained residues that could influence its subcellular localization, we examined whether this region in other STE family members might do the same. The first 200 aa of SopD2 have been shown to be required for its translocation into host cells, while the membraneassociation domain of this effector has been mapped to within the first 75 aa of the protein (Fig. 5a) (Brumell et al., 2003). Hence, both translocation and subcellular targeting domains are found within the same terminus of the protein. Partial protein sequence alignment of the $\mathrm{N}$-termini of STE 
(a)

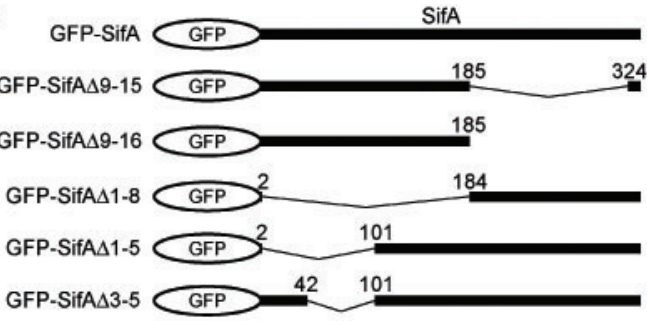
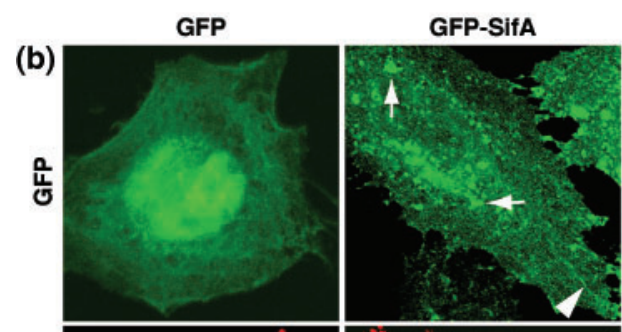

\section{GFP-SifA $\triangle 9-15$}

GFP-SifA $\triangle 9-16$
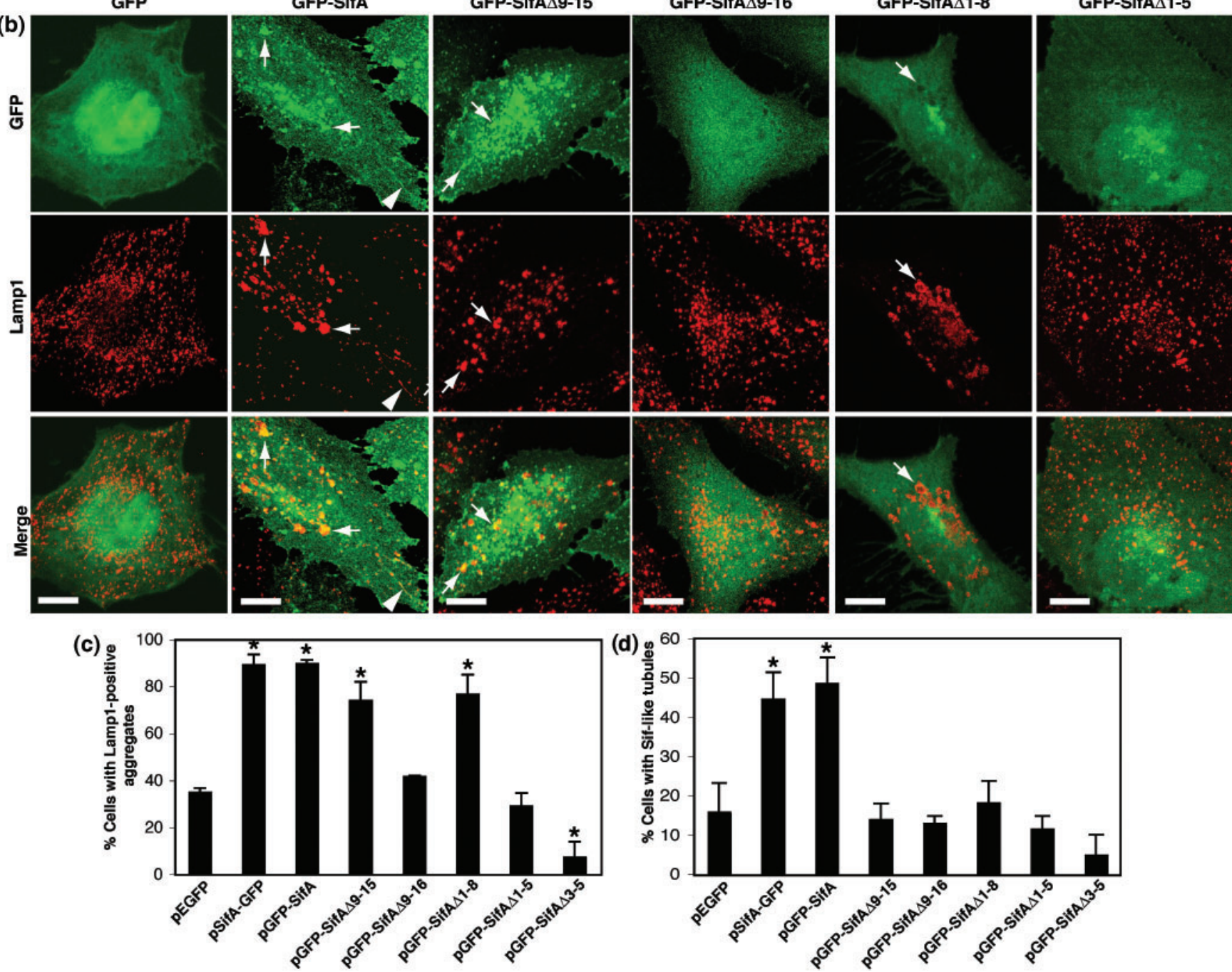

Fig. 3. Modulation of host cell endosome trafficking by SifA involves multiple domain activities. (a) Schematic of larger deletions constructed in GFP-SifA. Thin lines and numbers indicate positions of amino acids deleted in SifA. Full-length SifA is 336 aa. (b) Localization of GFP-SifA mutant proteins. HeLa cells were transfected with plasmids expressing the indicated GFP-SifA deletion mutant (green) for 16-20 h, fixed, immunostained with antibody to Lamp1 (red), and analysed by confocal microscopy. Bottom panels show the merge between corresponding GFP and Lamp1 signals, with colocalization indicated in yellow. Arrows indicate positions of swollen and/or aggregated Lamp $1^{+}$compartments. The arrowhead indicates Sif-like tubules. Size bars, $10 \mu \mathrm{m}$. (c). SifA domain functions on aggregation of Lamp ${ }^{+}$compartments. HeLa cells were transfected, fixed and stained as in (b) and the incidence of Lamp1-positive aggregates was determined. The results shown are means \pm SD of three separate experiments. Asterisks indicate $P<0 \cdot 05$. (d). SifA domain functions on Sif-like tubule formation. HeLa cells were transfected, fixed and stained as in (b) and the percentage of cells exhibiting Sif-like tubules was determined. The results shown are means \pm SD of three separate experiments. Asterisks indicate $P<0.05$. 

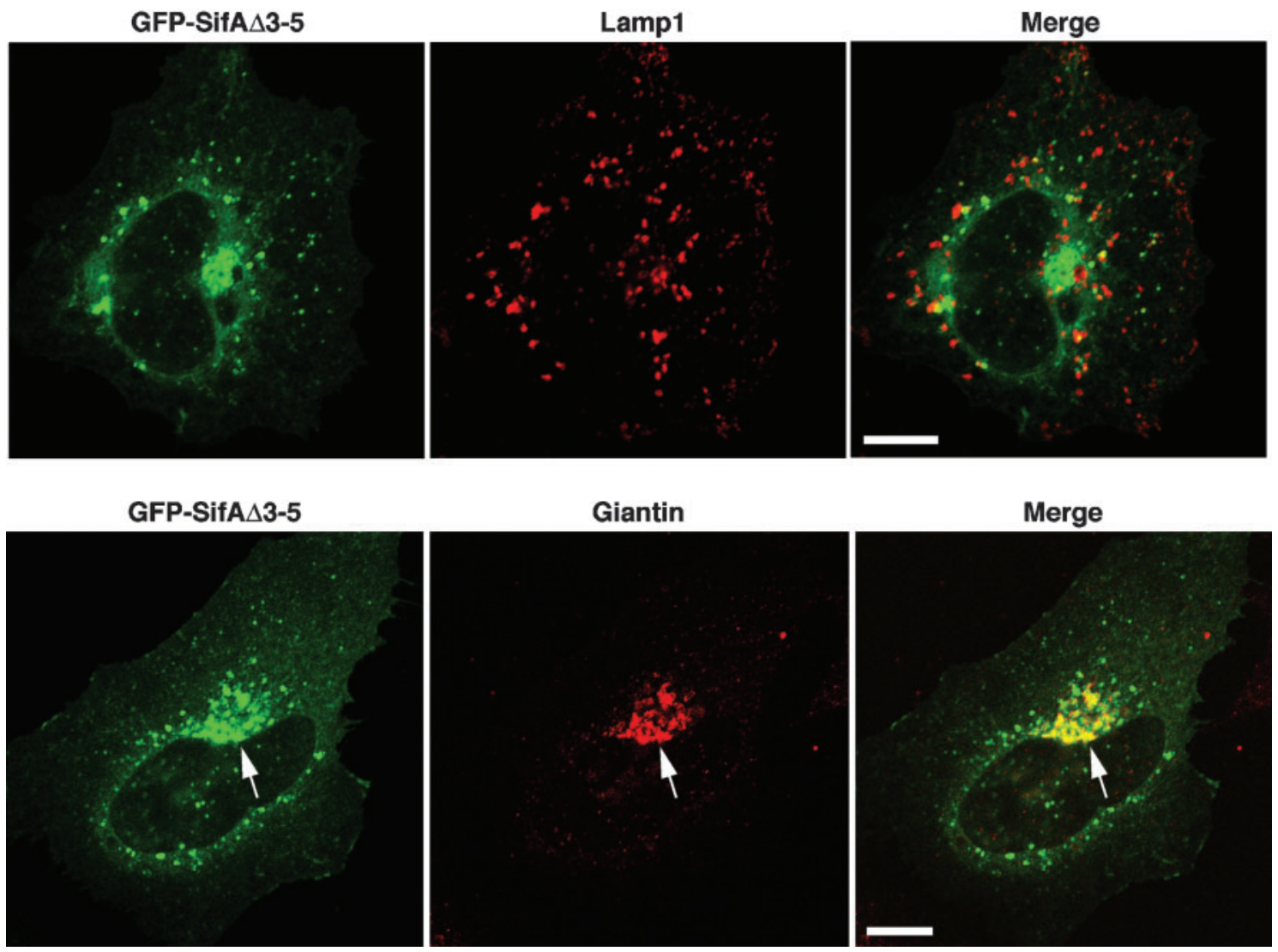

Fig. 4. A SifA truncation mutant can target the Golgi. HeLa cells were transfected with a plasmid expressing GFP-SifA $\Delta 3-5$ (See Fig. 2a for details) for 16-20 h, fixed, immunostained with antibody to Lamp1 (red, top panels) or to the Golgi protein giantin (red, bottom panels) and analysed by confocal microscopy. Arrows indicate colocalization of GFP-SifA $\Delta 3-5$ with giantin. Size bars, $10 \mu \mathrm{m}$.

family members SifA, SopD2, SseJ, SspH2 and SopD shows the conserved $\mathrm{WEK}(\mathrm{I} / \mathrm{M}) \mathrm{xxFF}$ motif previously identified as an important signal for effector translocation (Miao \& Miller, 2000). From the effectors used in our protein alignment, the consensus sequence of this conserved motif is $\mathrm{W}(\mathrm{E} / \mathrm{D})(\mathrm{K} / \mathrm{R}) \mathrm{xxxF}$; however, we will continue to use the previously established consensus of WEK(I/M)XxFF for discussion purposes (Miao \& Miller, 2000). Full-length protein sequence alignments (data not shown) indicate that the region encompassing this motif is one of the most conserved amongst STE members; hence we chose to examine this region in SopD2 (WDRFKDCF) (Fig. 5a) to determine its role, if any, in protein localization. GFP fusions to SopD2 bearing a complete deletion of its WEK(I/ $\mathrm{M}) \mathrm{xxFF}$ motif [SopD2( $\triangle \mathrm{aa} 37-44)-\mathrm{GFP}$ ] or simultaneous substitutions of W37P and F44R at highly conserved positions [SopD2(W37P,F44R)-GFP] were constructed and used in HeLa transfection studies. W37P and F44R substitutions were selected to disrupt a predicted helical conformation that spans the WEK(I/M)xxFF motif (Brumell et al., 2001a).

Confocal microscopy showed that wild-type SopD2-GFP colocalized with Lamp1 ${ }^{+}$vesicles (Fig. 6a, top panels), as previously observed (Brumell et al., 2003). In contrast, both SopD2( $\Delta$ aa37-44)-GFP and SopD2(W37P,F44R)-GFP appeared distributed throughout the cytosol and did not localize to Lamp1 ${ }^{+}$compartments (Fig. 6a, middle and bottom panels). Hence, residues within the conserved WEK(I/M)xxFF motif of SopD2 are required for membrane association to Lamp1 ${ }^{+}$compartments.

We next sought to determine whether the conserved STE region encompassing the WEK $(\mathrm{I} / \mathrm{M}) \mathrm{xxFF}$ motif had any inherent subcellular targeting properties. A fusion of GFP to SopD2 residues 31-64 (containing the peptide WDRFKDCF) (Fig. 5a) was constructed and transfected into HeLa cells. Transfected SopD2(aa31-64)-GFP did not colocalize with Lamp1 ${ }^{+}$vesicles, nor with SopD2-2HA translocated into the same cell by bacteria (Fig. 5b, bottom panels). Interestingly, SopD2(aa31-64)-GFP was concentrated to a juxtanuclear position (Fig. 5b, bottom left panel; Fig. 6b, top left panel), similar to GFP-SifA $\triangle 3-5$ (Fig. 4). However, the localization of SopD2(aa31-64)-GFP was much more distinct, and did not appear on other vesicles surrounding the nucleus as observed with GFP-SifA $\Delta 3-5$. The position of SopD2(aa31-64)-GFP suggested it targeted 


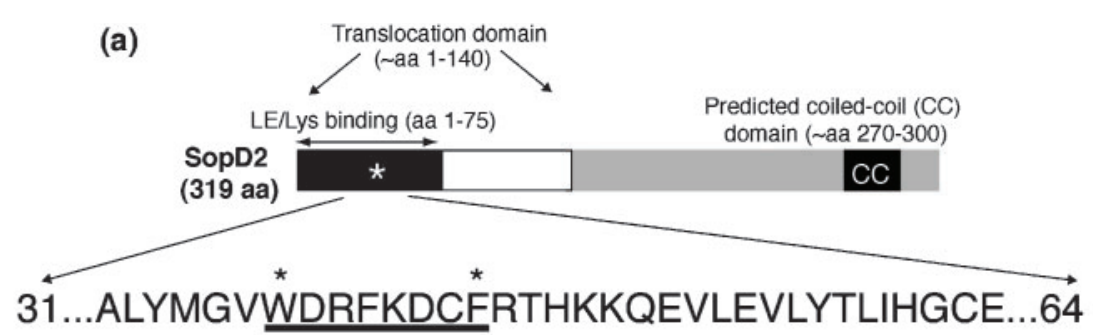

(b)
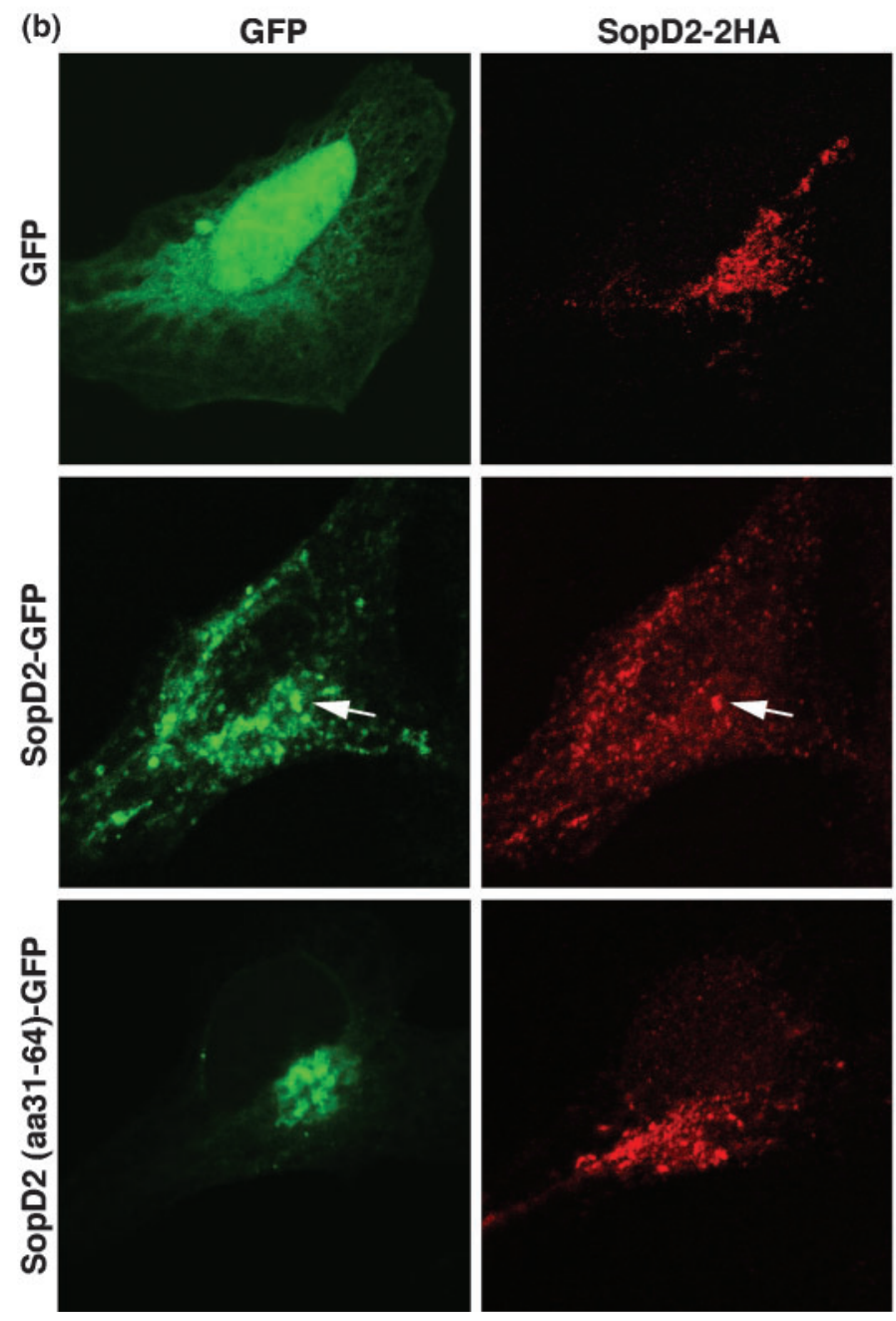
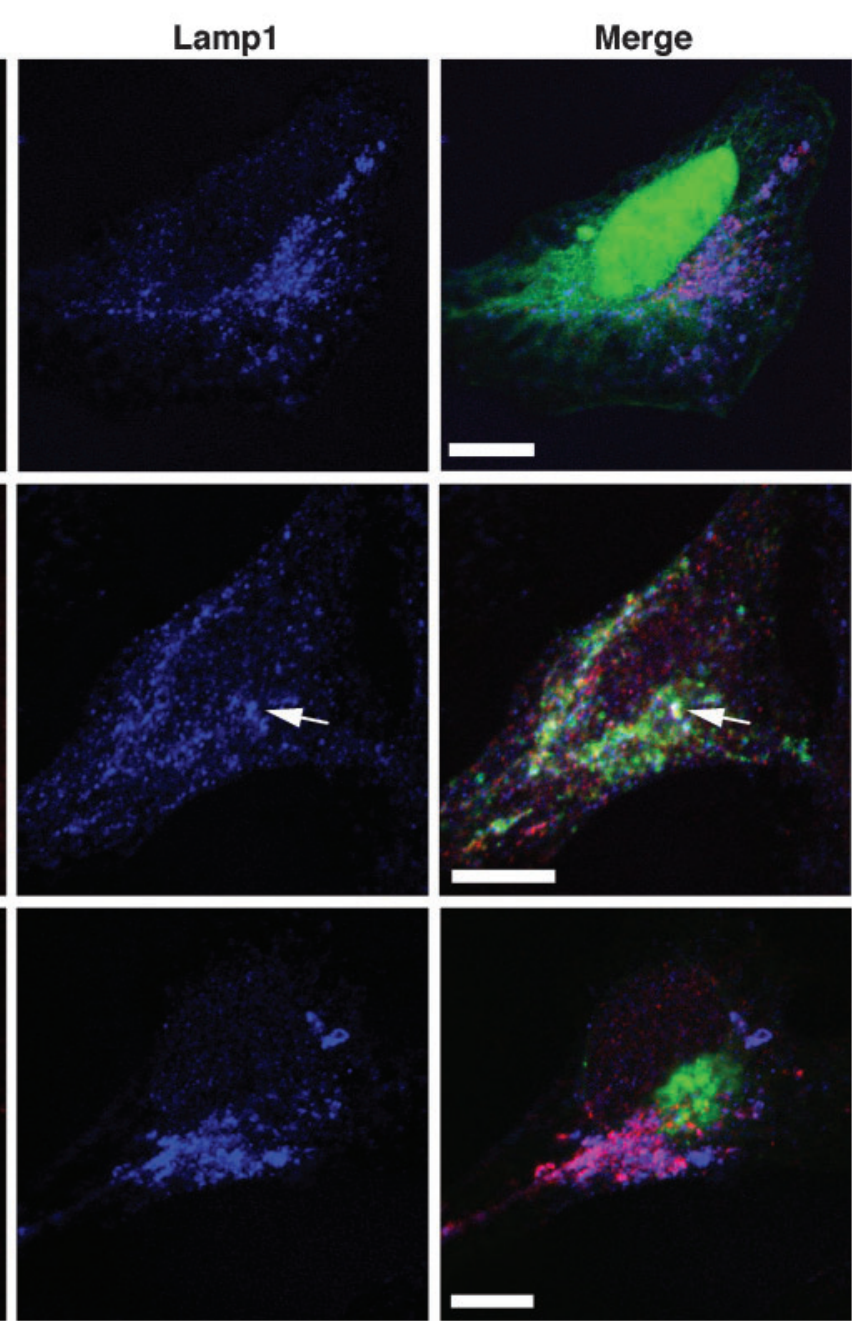

Fig. 5. SopD2 contains a conserved WEK(I/M)xxFF motif implicated in intracellular targeting. (a) The conserved WEK(I/ M)xxFF motif of SopD2 is underlined. Shown are residues 31-64 of SopD2, selected for fusion with GFP. Asterisks indicate conserved residues selected for further mutagenesis. (b) Transfected SopD2-GFP colocalizes with translocated SopD2-2HA and Lamp1-positive compartments, but SopD2(aa31-64)-GFP does not. HeLa cells were transfected with plasmids expressing GFP alone (top panels), or GFP-fusions to wild-type SopD2 (middle panels) or SopD2(aa31-64) (bottom panels) for 16-20 h. Cells were then infected with a $\Delta s o p D 2$ strain of serovar Typhimurium SL 1344 containing a plasmid encoding SopD2-2HA. After $8 \mathrm{~h}$ of infection, cells were fixed, permeabilized, and co-immunostained with antibodies to HA-tag (red) and Lamp1 (blue). Images show flat projections of assembled confocal sections. Pink signals indicate colocalization of translocated SopD2-HA and Lamp1 signals. White signals indicate colocalization of translocated SopD2-HA, transfected SopD2-GFP and Lamp1 (arrow). Size bars, $10 \mu \mathrm{m}$.

the Golgi, which was confirmed by its extensive colocalization with the Golgi markers giantin (Fig. 6b, top panels) and GM130 (data not shown). Treatment of HeLa cells expressing SopD2(aa31-64)-GFP with the Golgi-disrupting drug BFA resulted in the dispersal of fluorescent signal throughout the cytosol (Fig. 6c). The effects of BFA were 


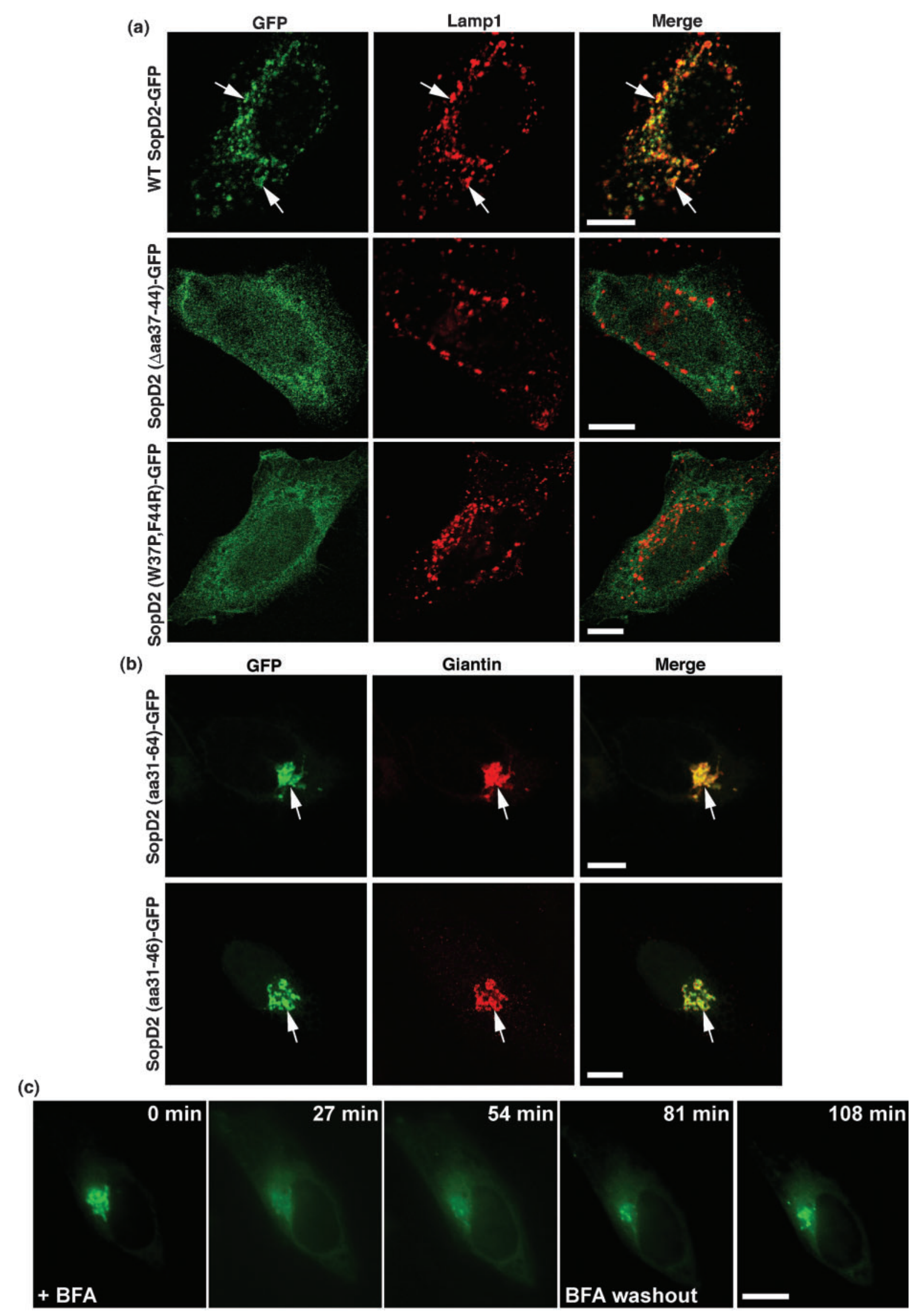


reversible, with SopD2(aa31-64)-GFP signal returning to its original position following washout of the drug (Fig. 6c). A GFP fusion to a smaller SopD2 peptide composed of only 16 aa (aa 31-46) and retaining the residues WDRFKDCF was also sufficient to specifically target the Golgi, as shown by its colocalization with giantin (Fig. 6b, bottom panels). SopD2(aa31-46)-GFP also did not colocalize with Lamp1 ${ }^{+}$ compartments (data not shown), similar to SopD2(aa3164)-GFP. Overall, these results show that residues within the conserved WEK(I/M)xxFF motif of SopD2 are involved in directing this STE family member to endocytic vesicles. Furthermore, SopD2 peptides containing this conserved motif can target the Golgi.

\section{A conserved $\mathbf{N}$-terminal peptide present in a subset of STE effectors can target the Golgi}

To determine the subcellular localization characteristics of WEK $(\mathrm{I} / \mathrm{M}) \mathrm{xxFF}$ motif-containing peptides from other STE family members, GFP-fusions to such peptides from SseJ, SspH2, SopD2 and SifA were constructed (Fig. 7). Like SifA, the localization of each of these STE effectors upon transfection has already been established. SseJ localizes to globular membranous Lamp ${ }^{+}$compartments that have a composition similar to that of SCVs (Ruiz-Albert et al., 2002), SspH2 associates with regions of active actin polymerization such as membrane ruffles (Miao et al., 2003), and SopD, a SPI-1 effector, appears to be cytosolically distributed (Brumell et al., 2003).

Transfection of HeLa cells with SspH2(aa28-61)-GFP and SseJ(aa28-61)-GFP resulted in a noticeable fraction of each fusion localizing to an area adjacent to the nucleus, with the remaining protein diffusely distributed throughout the cytosol (Fig. 7a, b). Colocalization with giantin confirmed that SspH2 (aa28-61)-GFP and SseJ(aa28-61)-GFP could be targeted to the Golgi (Fig. 7a, b), although not to the same extent as SopD2(aa31-64)-GFP (Fig. 6b). Interestingly, SopD(aa31-64)-GFP was found nearly exclusively within the nucleus (Fig. 7c). Although some of the fusion was also observed in a region next to the nucleus, it did not exhibit significant colocalization with the Golgi marker giantin (Fig. 7c). SifA(aa25-58)-GFP was distributed in a network surrounding the nucleus, with only limited colocalization with giantin (Fig. 7d). Much of the SifA(aa25-58)-GFP signal was extended beyond that of giantin; however, its perinuclear distribution did not significantly colocalize with an endoplasmic reticulum marker, protein disulphide isomerase (data not shown).

Although both SifA(aa25-58)-GFP and GFP-SifA $\Delta 3-5$ contained the conserved WEK(I/M)xxFF motif, we noted that they each had a distinct localization upon transfection (compare Figs 4 and 7d). This suggested that, while the WEK(I/M)xxFF can function to direct subcellular targeting, the overall context of the protein in which this motif appears may play an important role in influencing its ultimate localization. In support of this, GFP-SifA $\Delta 1$, with amino acids $2-21$ of SifA deleted but retaining the conserved WEK(I/M)xxFF motif, localized exclusively to the nucleus upon transfection and was not colocalized with any Lamp $1^{+}$compartments (Fig. 7e). Overall, our results show that a subset of STE effectors contain N-terminal motifs that can target the Golgi. Furthermore, mutations such as deletions may liberate other cryptic subcellular localization motifs in bacterial effectors.

\section{DISCUSSION}

This is believed to be the first study to examine the distribution of functional domains in the STE family member SifA. To exert its effects, SifA must be secreted through the SPI-2 T3SS, engage and tubulate late endocytic compartments, and interact with specific regulators of microtubule motors (Beuzon et al., 2000; Boucrot et al., 2005; Brumell et al., 2001a, b, 2002; Harrison et al., 2004; Stein et al., 1996). As such, it is expected that several functional domains would exist within this effector to regulate its activities. Evidence for this can be seen with the SifA-2HA construct used in our studies. This fusion consists of a 20 aa insertion (encoding tandem HA epitopes) following amino acid 136 of wild-type SifA (Brumell et al., 2002). That this protein is translocated efficiently and permits a $\Delta$ sifA mutant to induce Sif formation suggested, in previous studies, that SifA consists of at least two domains approximated by $\mathrm{N}$ - and $\mathrm{C}$-terminal halves that could be separated by the $2 \mathrm{HA}$ insertion (Brumell et al., 2002).

To date, the best-characterized SifA region is its membraneanchoring C-terminus, containing a 6 aa sequence with homology to CAAX and Rab geranylgeranyl transferase prenylation motifs (Boucrot et al., 2003; Reinicke et al., 2005). Biochemical studies have shown that this motif is a site for isoprenoid attachment, with an adjacent cysteine residue modified by S-acylation (Reinicke et al., 2005).

Fig. 6. A conserved N-terminal motif in SopD2 is involved in membrane association and is sufficient to target the Golgi. (a) HeLa cells were transfected with plasmids expressing the indicated SopD2-GFP mutants (green) for 16-20 h, fixed, and immunostained with antibody to Lamp1 (red). Right panels show the merge between corresponding GFP and Lamp1 signals, with colocalization indicated in yellow. Arrows indicate colocalization of SopD2-GFP with Lamp1 ${ }^{+}$compartments. Size bars, $10 \mu \mathrm{m}$. (b) HeLa cells transfected with plasmids expressing fusions of SopD2 peptides (aa31-64 or aa31-46) to GFP for 16-20 h, fixed, and immunostained with antibody to giantin (red). Arrows indicate colocalization of SopD2-GFP with giantin. Size bars, $10 \mu \mathrm{m}$. (c). HeLa cells expressing GFP-SopD2(aa31-64) were treated with the Golgi-disrupting drug BFA and observed using live cell imaging. BFA was removed after $70 \mathrm{~min}$ and cells were allowed to recover in drug-free medium. Size bar, $10 \mu \mathrm{m}$. 

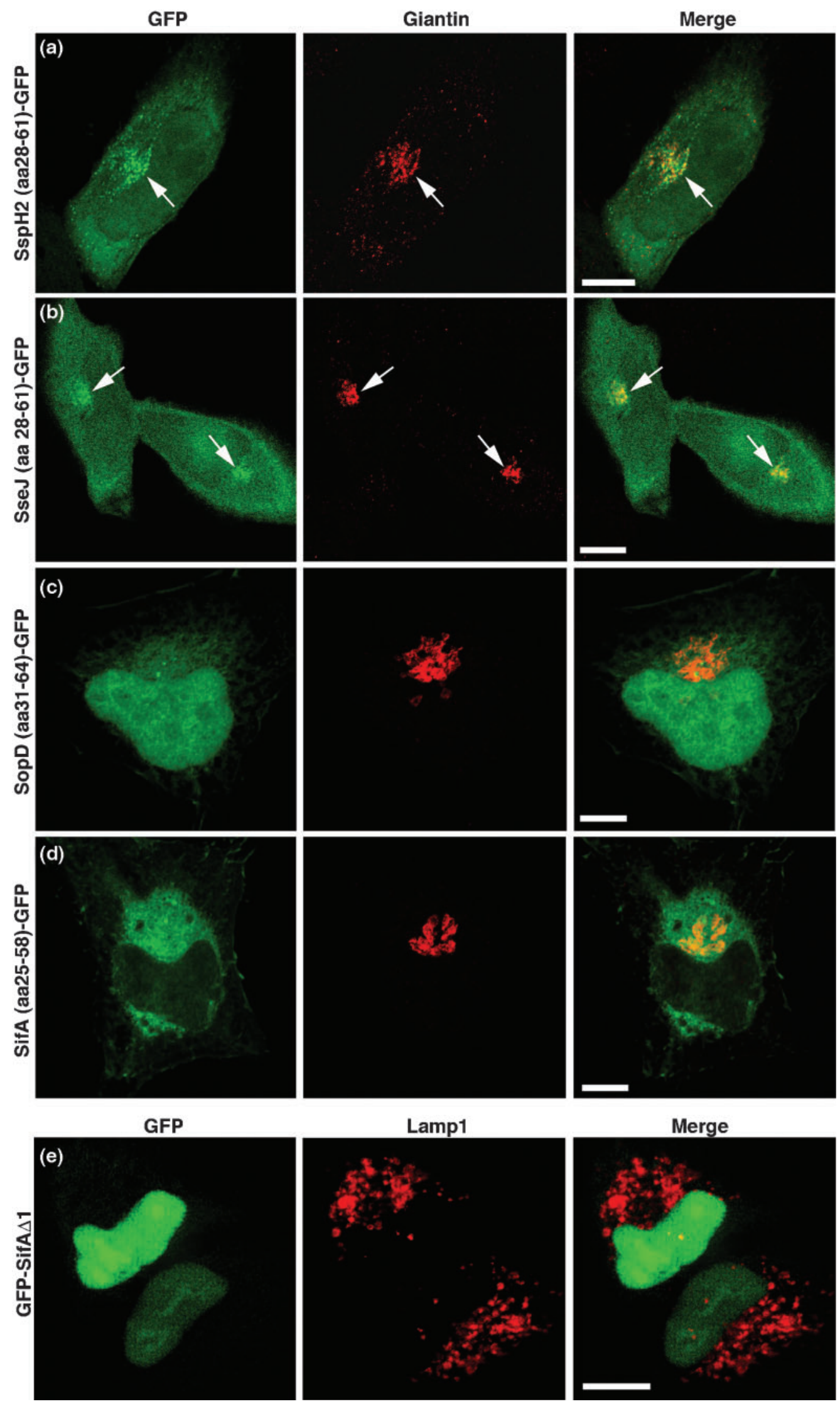

Lamp1

Merge
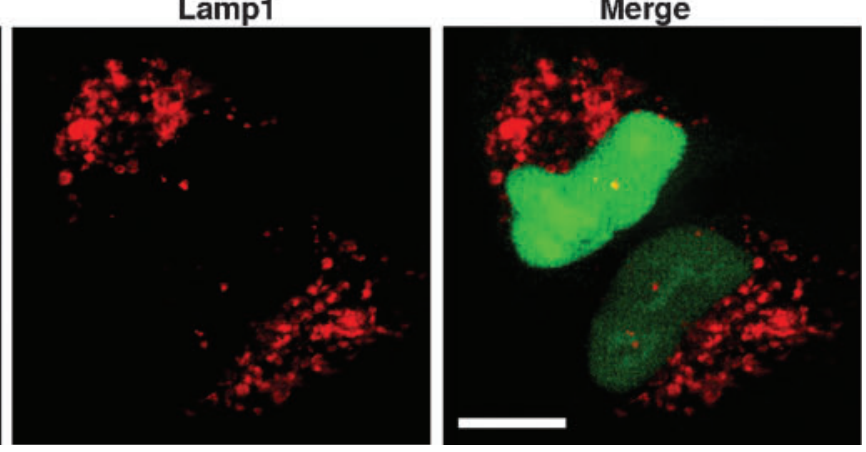
Fusion of an 11 aa C-terminal SifA sequence containing this CAAX motif is sufficient to target a GFP fusion to uncharacterized membranes upon transfection of HeLa cells (Boucrot et al., 2003). Our results extend the above studies and demonstrate that the N-terminal half of SifA is sufficient to mediate targeting and aggregation of Lamp1 ${ }^{+}$compartments, provided it is fused to the SifA prenylation motif. Moreover, our results also show that the C-terminal half of SifA containing the membrane-anchoring motif is not sufficient for localizing the effector to Lamp $1^{+}$compartments. This was evident with GFP-SifA $\Delta 1-5$ and GFPSifA $\Delta 3-5$, each having reduced association with Lamp1 ${ }^{+}$ vesicles despite retaining the $\mathrm{C}$-terminal membrane anchor. This indicates that SifA targeting to appropriate host cell compartments is a combined result of membrane anchoring via prenylation and by other regions distributed throughout SifA, including determinants found within the N-terminus. Collectively, this would provide an effective concentration of SifA at the site of action and induce the aggregation of Lamp $1^{+}$compartments.

GFP-SifA truncation derivatives that retained the ability to aggregate Lamp1 $^{+}$vesicles could not tubulate these compartments, in contrast to unmodified GFP-SifA. Thus both $\mathrm{N}$ - and C-terminal domains of SifA are required for the formation of Sif-like tubules. To date, several studies have linked SifA effector function to mediating interactions with microtubule motors and/or motor adaptors to control the membrane dynamics of SCVs and/or Sifs (Boucrot et al., 2005; Guignot et al., 2004; Harrison et al., 2004). Therefore the effector domain(s) of SifA that regulate these activities may span regions encompassing the entire protein, as compared to the more discrete C-terminal effector domains found in other translocated bacterial proteins (Hueck, 1998).

Our studies also demonstrate that a completely intact SifA is required for its proper secretion and translocation. SifA translocation appears to involve regions in addition to those described for other effectors. Deletions in the N-terminal 140 aa disrupted translocation, consistent with SifA possessing a proteinaceous $\mathrm{N}$-terminal or $5^{\prime} \mathrm{mRNA}$-encoded secretion signal and a chaperone-dependent signal (Ramamurthi \& Schneewind, 2003). However, our results are also consistent with regions downstream of amino acid 140 being required for SifA translocation, lying beyond the $\mathrm{N}$-terminal translocation domain common to the STE family (Miao \& Miller, 2000). Other bacterial effectors have been characterized to have translocation signals localized in their C-termini, including the serovar Typhimurium SPI-1 effector SipC (Chang et al., 2005) and translocated intimin receptor (Tir) from enterohaemorrhagic E. coli (EHEC O157:H7) (Allen-Vercoe et al., 2005).

Previous results have shown that various truncated SifA fusions to CyaA were not translocated into host cells and tended to be unstable (Miao \& Miller, 2000). For the most part, the deletions used in our study did not appear to negatively affect SifA-2HA levels in serovar Typhimurium; hence, the differences in secretion compared to wild-type protein were not likely due to poor protein synthesis or stability. Although translocation into host cells was disrupted, we note that there was still some detectable secretion of several mutant proteins into the growth medium. The continued secretion of SifA-2HA $\Delta 2$ indicates that the WEK(I/M)xxFF is not required for SifA secretion. The secretion of an effector is not indicative of its ability to be translocated into a host cell, suggesting that other factors are needed. This is evidenced by YopE and YopH effector proteins from Yersinia species. While YopE and YopH secretion signals were mapped within the first 15 and 17 residues of each respective protein, additional residues encompassing the first 50 and 71 residues of YopE and YopH, respectively, were needed for their translocation into host cells (Sory et al., 1995). Hence, residues in addition to those directing secretion may be required to properly interact with specific chaperones and/or the translocon pore to efficiently direct effector translocation into a host cell. It is also possible that certain SifA-2HA mutants were secreted through the flagellar apparatus, as described previously with deletion mutants in serovar Typhimurium effectors SopE and SptP (Lee \& Galan, 2004). As translocation is dependent on a T3SS apparatus, the secretion of an effector through a flagellar apparatus would therefore not result in its delivery into a host cell.

Residues within the conserved WEK(I/M)xxFF motif have been shown to be essential for translocation of various STE family members (Miao \& Miller, 2000). We provide evidence that this motif has at least two roles, in both translocation and protein localization in host cells. Using SopD2 as a model STE effector, we demonstrated that either deletion or mutation of residues in the conserved $\mathrm{N}$-terminal WEK(I/M)xxFF motif abrogates its localization to late endocytic compartments. Significantly, this appears to be the first report to identify specific residues within a short, defined translocation domain that can direct subcellular localization. Other studies have shown a relationship between translocation domains and subcellular targeting

Fig. 7. A conserved N-terminal peptide present in $\mathrm{SspH} 2$ and SseJ can target the Golgi. HeLa cells were transfected with plasmids expressing GFP-fusions to a conserved N-terminal region found in various serovar Typhimurium effectors, including (a) SspH2(aa28-61), (b) SseJ(aa28-61), (c) SopD(aa31-64), and (d) SifA(aa25-58). At 16-20 h after transfection, cells were fixed and immunostained with antibodies to giantin (red). Right panels in $(a-d)$ show the merge between corresponding GFP and giantin signals, with colocalization indicated in yellow. Arrows indicate colocalization of SspH2(aa28-61)-GFP and SseJ(aa28-61)-GFP with giantin. Size bars, $10 \mu \mathrm{m}$. (e) Nuclear localization of GFP-SifA $\Delta 1$. HeLa cells were transfected with a GFP-SifA $\Delta 1$ expressing plasmid for 16-20 h, fixed, and immunostained with antibody to Lamp1 (red). The right panel shows the merge between corresponding GFP and Lamp1 signals. 
(Knodler et al., 2003; Miao et al., 2003); however, those studies focused on relatively large protein regions $(>150$ residues) and did not identify specific residues involved in protein localization. Our results reinforce the notion that small regions in bacterial effectors can mediate multiple activities. Such multifunctional domains have been observed in other bacterial effector proteins, including the 80 residue $\mathrm{N}$-terminus of the Shigella flexneri T3SS substrate IpaC, where the secretion signal, chaperone-binding region, IpaBbinding domain and IpaC invasion function are all encoded (Harrington et al., 2003). More recently, a small C-terminal peptide region (LFNEF) within the non-STE effector PipB2 was identified to be important for both targeting and function of the effector on late endocytic and lysosomal compartments (Knodler \& Steele-Mortimer, 2005). We note that the SopD2( $\Delta$ aa37-44)-GFP fusion did appear to induce some aggregation of Lamp1 ${ }^{+}$compartments in a small fraction of transfected cells. Hence, some function may be retained despite the inability of the mutant fusion protein to overtly co-localize with these compartments. It is possible that the overexpression of SopD2( $\Delta$ aa37-44)-GFP can partially overcome its inability to specifically co-localize with Lamp $1^{+}$compartments, and induce their aggregation in some transfected cells.

Curiously, peptides containing the WEK(I/M)xxFF motif from a subset of STE effectors, particularly SopD2, are targeted to the Golgi apparatus upon transfection. Ectopic expression of the non-STE effector SseG results in its association with the Golgi as well (Salcedo \& Holden, 2003). A 55 aa region predicted to be a transmembrane domain has been identified as the SseG Golgi-targeting region (Salcedo \& Holden, 2003); however, protein sequence alignments show there is no similarity between this region and the WEK(I/M)xxFF-containing peptides used in our study (Fig. 8). The biological significance, if any, of Golgi targeting by $\mathrm{WEK}(\mathrm{I} / \mathrm{M}) \mathrm{xxFF}$-containing peptides is not known. It is possible that certain STE effectors are first directed to the secretory pathway before redistribution to other cell regions, but such an intermediate localization step has not been observed. Numerous proteins residing in the endosomal system transit through the endoplasmic reticulum and Golgi; however, identifying these intermediates by methods such as immunofluorescence can be difficult (Cook et al., 2004). Since SifA is modified by prenylation and $S$-acylation, it is possible that this effector may be processed through the endoplasmic reticulum (Reinicke et al., 2005). Hence, it is conceivable that certain effectors may need to pass through the host cell secretory pathway before interacting with their final intracellular targets.

We note that the conserved WEK(I/M)xxFF motifs have similarity to a previously characterized phosphatidic acid (PA)-binding domain found in PDE4A1 cAMP-specific phosphodiesterase implicated in Golgi targeting (Baillie et al., 2002). Golgi-specific localization of PDE4A1 is likely a result of binding to enriched PA formed from phospholipase D localized in the Golgi (Baillie et al., 2002; Freyberg et al., 2001, 2003). The PDE4A1 peptide region, referred to as TAPAS-1 (tryptophan anchoring phosphatidic acid selective binding domain 1), is an 11 aa sequence proposed to form an amphipathic $\alpha$-helix that interacts with PA-containing surfaces by insertion of the peptide in a parallel orientation to membrane surfaces (Baillie et al., 2002). Fusion of a 25 aa TAPAS-1-containing fragment to GFP was shown to target the protein almost exclusively to the Golgi (Baillie et al., 2002), similar to our observations with SopD2 peptides containing their cognate WDRFKDCF motif. Strikingly, sequence alignment reveals that the SopD2 WEK(I/M)xxFF motif (sequence WDRFKDCF) shares four identical residues (bold) and two polar/charged residues (underlined) with the TAPAS-1 microdomain (PWLVGWWDQFKR) (Fig. 8). However, preliminary studies probing phosphoinositideand PA-spotted nitrocellulose strips with a GST fusion to the SopD2 Golgi-targeting peptide have not shown specific binding to PA under the conditions tested.

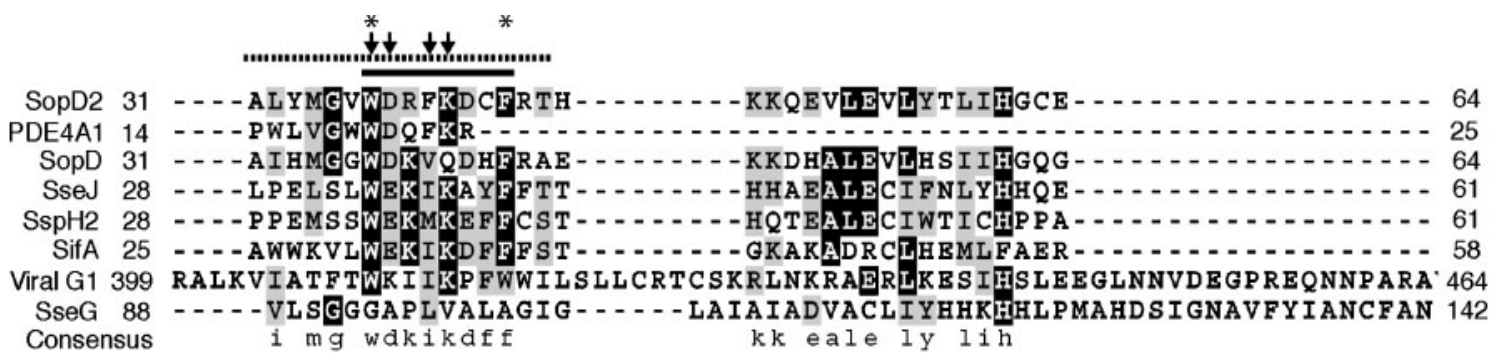

Fig. 8. Partial CLUSTAL W multiple alignment of STE proteins SopD2, SopD, SseJ, SspH2 and SifA showing the conserved WEK(I/M)xxFF motif (residues under black bar). Asterisks indicate conserved W37 and F44 residues found in the WEK(I/ M)xxFF motif. The STE family member residues shown were all fused to GFP for localization studies. Residues underneath the dotted line correspond to SopD2 residues 31-46. Also aligned are the Golgi-targeting sequences determined for serovar Typhimurium effector SseG (Salcedo \& Holden, 2003) and Uukuniemi virus (Bunyaviridae) glycoprotein G1 (Andersson \& Pettersson, 1998), as well as the phosphatidic acid (PA)-binding domain of rat PDE4A1 cAMP-specific phosphodiesterase (Baillie et al., 2002). Arrows indicate identical residues between the Golgi-targeting motif of SopD2 and the PA-binding domain of PDE4A1. 
Interestingly, a region within the Golgi-targeting Cterminus of Uukuniemi virus G1 membrane glycoprotein (Andersson \& Pettersson, 1998) shares some similarity with both TAPAS-1 and WEK(I/M)xxFF motifs (Fig. 8; residues underneath black bar). GFP fusions to viral G1 peptides (as small as 30 residues) containing the region resembling the $\mathrm{WEK}(\mathrm{I} / \mathrm{M}) \mathrm{xxFF}$ consensus have also been shown to target the Golgi (Andersson \& Pettersson, 1998). That bacterial and viral proteins may have evolved to contain similar TAPAS-1-like sequences to host proteins is intriguing, and may present evidence of convergent evolution to exploit various host cell systems. The specificity of TAPAS-1-like peptides for the Golgi, including the SopD2 peptides used in the present study, might serve as a useful cell biology tool in future studies requiring Golgi localization.

It remains possible that the distinct localization patterns we observed with our constructs may have resulted from the liberation of cryptic subcellular targeting signals by deletion of surrounding residues that would normally mask or override them. For example, our GFP-SifA $\Delta 1$ mutant was localized to the nucleus, a compartment where SifA is not normally observed. Nuclear localization has also been observed with a GFP-SifA mutant lacking its membraneanchoring motif (Boucrot et al., 2003). Thus, in the context of the present study, the Golgi and nuclear-specific targeting of SopD2 and SifA peptides, respectively, may represent artefacts due to drastic deletions. In addition, the method of introducing effectors into host cells can influence protein localization. This is evidenced by the Golgi-specific localization of transfected SseG compared to its association with SCV and Sifs when translocated by bacteria (Salcedo \& Holden, 2003). Furthermore, it is possible that some deletions may cause protein misfolding that can affect effector protein function and localization. Hence, the overall context of the effector and how it is presented to the host cell, including the nature of deletions or mutations, the presence of other effectors, the quantity of effector protein present in the cell and its delivery by bacterial translocation or by transfection, may influence the targeting of the protein to possibly produce artefacts, and should be carefully considered when engaging in bacterial effector protein studies.

Overall, we have identified regions of SifA required for various aspects of its biological function. It is apparent that regions distributed throughout SifA are required for secretion and translocation. We provide evidence that both $\mathrm{N}$ - and C-terminal halves of SifA play roles in mediating the binding and aggregation of Lamp $1^{+}$vesicles. However, neither domain was sufficient to induce the formation of Sif-like tubules. Thus, SifA is composed of $\mathrm{N}$ - and C-terminal domains that must act cooperatively to effectively tubulate late endocytic compartments. As such, this may make precise structure-function assignments to SifA more challenging than for other effectors. SopD2 also appears to contain a multifunctional $\mathrm{N}$-terminus that mediates both translocation and subcellular localization. Significantly, we show that residues in the conserved STE family WEK(I/M)xxFF motif are involved in subcellular targeting by SopD2. Hence, discrete translocation domains can also function in protein localization. Finally, we show that peptides present in a subset of STE family members can target discrete subcellular locations, suggesting that cryptic binding motifs within bacterial effectors can be liberated depending on the context of their presentation within the host cell.

\section{ACKNOWLEDGEMENTS}

This work was supported by grants to B. B.F. from the Canadian Institutes of Health Research (CIHR) and the Howard Hughes Medical Institute International Scholar Program. B. B. F. is a CIHR Distinguished Investigator, a HHMI International Research Scholar and the University of British Columbia Peter Wall Distinguished Professor. This work was also supported by grant funding and a New Investigator Award from CIHR to J. H. B. Infrastructure for the Brumell laboratory was provided by a New Opportunities Fund from the Canadian Foundation for Innovation and the Ontario Innovation Trust. John H. Brumell, $\mathrm{PhD}$ holds an Investigators in Pathogenesis of Infectious Diseases Award from the Burroughs Wellcome Fund. J.H.B is a recipient of the Premier's Research Excellence Award from the Ontario Ministry of Economic Development and Trade, and a Boehringer Ingelheim (Canada) Young Investigators Award in Biological Sciences. B. K. C is the recipient of a CIHR postdoctoral fellowship and a Michael Smith Foundation for Health Research fellowship. J. S. is supported by a CAG/CIHR/Solvay Pharma postdoctoral fellowship administered by the Canadian Association of Gastroenterology. N. F. B. is a recipient of a Michael Smith Foundation for Health Research fellowship. We thank members of the Brumell and Finlay laboratory for critical reading of this manuscript. We are grateful to Dr Charles Deber and Arianna Rath, Hospital for Sick Children, for help with analysis of predicted peptide structures.

\section{REFERENCES}

Allen-Vercoe, E., Toh, M. C., Waddell, B., Ho, H. \& DeVinney, R. (2005). A carboxy-terminal domain of Tir from enterohemorrhagic Escherichia coli O157:H7 (EHEC O157:H7) required for efficient type III secretion. FEMS Microbiol Lett 243, 355-364.

Anderson, D. M. \& Schneewind, O. (1997). A mRNA signal for the Type III secretion of Yop proteins by Yersinia enterocolitica. Science 278, 1140-1143.

Andersson, A. M. \& Pettersson, R. F. (1998). Targeting of a short peptide derived from the cytoplasmic tail of the G1 membrane glycoprotein of Uukuniemi virus (Bunyaviridae) to the Golgi complex. J Virol 72, 9585-9596.

Baillie, G. S., Huston, E., Scotland, G. \& other authors (2002). TAPAS-1, a novel microdomain within the unique N-terminal region of the PDE4A1 cAMP-specific phosphodiesterase that allows rapid, $\mathrm{Ca}^{2+}$-triggered membrane association with selectivity for interaction with phosphatidic acid. J Biol Chem 277, 28298-28309.

Bakshi, C. S., Singh, V. P., Wood, M. W., Jones, P. W., Wallis, T. S. \& Galyov, E. E. (2000). Identification of SopE2, a Salmonella secreted protein which is highly homologous to SopE and involved in bacterial invasion of epithelial cells. J Bacteriol 182, 2341-2344.

Beuzon, C. R., Banks, G., Deiwick, J., Hensel, M. \& Holden, D. W. (1999). pH-dependent secretion of SseB, a product of the SPI-2 type III secretion system of Salmonella typhimurium. Mol Microbiol 33, 806-816. 
Beuzon, C. R., Meresse, S., Unsworth, K. E., Ruiz-Albert, J., Garvis, S., Waterman, S. R., Ryder, T. A., Boucrot, E. \& Holden, D. W. (2000). Salmonella maintains the integrity of its intracellular vacuole through the action of SifA. EMBO J 19, 3235-3249.

Beuzon, C. R., Salcedo, S. P. \& Holden, D. W. (2002). Growth and killing of a Salmonella enterica serovar Typhimurium sifA mutant strain in the cytosol of different host cell lines. Microbiology 148, 2705-2715.

Birmingham, C. L., Jiang, X., Ohlson, M. B., Miller, S. I. \& Brumell, J. H. (2005). Salmonella-induced filament formation is a dynamic phenotype induced by rapidly replicating Salmonella enterica serovar typhimurium in epithelial cells. Infect Immun 73, 1204-1208.

Boucrot, E., Beuzon, C. R., Holden, D. W., Gorvel, J. P. \& Meresse, S. (2003). Salmonella typhimurium SifA effector protein requires its membrane-anchoring C-terminal hexapeptide for its biological function. J Biol Chem 278, 14196-14202.

Boucrot, E., Henry, T., Borg, J. P., Gorvel, J. P. \& Meresse, S. (2005). The intracellular fate of Salmonella depends on the recruitment of kinesin. Science 308, 1174-1178.

Brumell, J. H. \& Grinstein, S. (2004). Salmonella redirects phagosomal maturation. Curr Opin Microbiol 7, 78-84.

Brumell, J. H., Marcus, S. L. \& Finlay, B. B. (2000). N-terminal conservation of putative type III secreted effectors of Salmonella typhimurium. Mol Microbiol 36, 773-774.

Brumell, J. H., Rosenberger, C. M., Gotto, G. T., Marcus, S. L. \& Finlay, B. B. (2001a). SifA permits survival and replication of Salmonella typhimurium in murine macrophages. Cell Microbiol 3, 75-84.

Brumell, J. H., Tang, P., Mills, S. D. \& Finlay, B. B. (2001b). Characterization of Salmonella-induced filaments (Sifs) reveals a delayed interaction between salmonella-containing vacuoles and late endocytic compartments. Traffic 2, 643-653.

Brumell, J. H., Goosney, D. L. \& Finlay, B. B. (2002). SifA, a type III secreted effector of Salmonella typhimurium, directs Salmonellainduced filament (Sif) formation along microtubules. Traffic 3, 407-415.

Brumell, J. H., Kujat-Choy, S., Brown, N. F., Vallance, B. A., Knodler, L. A. \& Finlay, B. B. (2003). SopD2 is a novel type III secreted effector of Salmonella typhimurium that targets late endocytic compartments upon delivery into host cells. Traffic 4, 36-48.

Chakravortty, D., Hansen-Wester, I. \& Hensel, M. (2002). Salmonella pathogenicity island 2 mediates protection of intracellular Salmonella from reactive nitrogen intermediates. J Exp Med 195, 1155-1166.

Chang, J., Chen, J. \& Zhou, D. (2005). Delineation and characterization of the actin nucleation and effector translocation activities of Salmonella SipC. Mol Microbiol 55, 1379-1389.

Cirillo, D. M., Valdivia, R. H., Monack, D. M. \& Falkow, S. (1998), Macrophage-dependent induction of the Salmonella pathogenicity island 2 type III secretion system and its role in intracellular survival. Mol Microbiol 30, 175-188.

Cook, N. R., Row, P. E. \& Davidson, H. W. (2004). Lysosome associated membrane protein 1 (Lamp1) traffics directly from the TGN to early endosomes. Traffic 5, 685-699.

Coombes, B. K., Brown, N. F., Kujat-Choy, S., Vallance, B. A. \& Finlay, B. B. (2003). SseA is required for translocation of Salmonella pathogenicity island-2 effectors into host cells. Microbes Infect 5, 561-570.

Coombes, B. K., Brown, N. F., Valdez, Y., Brumell, J. H. \& Finlay, B. B. (2004). Expression and secretion of Salmonella pathogenicity island-2 virulence genes in response to acidification exhibit differential requirements of a functional type III secretion apparatus and SsaL. J Biol Chem 279, 49804-49815.
Cornelis, G. R. \& Van Gijsegem, F. (2000). Assembly and function of type III secretory systems. Annu Rev Microbiol 54, 735-774.

Dunlap, N. E., Benjamin, W. H., Jr, McCall, R. D., Jr, Tilden, A. B. \& Briles, D. E. (1991). A 'safe-site' for Salmonella typhimurium is within splenic cells during the early phase of infection in mice. Microb Pathog 10, 297-310.

Freyberg, Z., Sweeney, D., Siddhanta, A., Bourgoin, S., Frohman, M. \& Shields, D. (2001). Intracellular localization of phospholipase D1 in mammalian cells. Mol Biol Cell 12, 943-955.

Freyberg, Z., Siddhanta, A. \& Shields, D. (2003). "Slip, sliding away": phospholipase D and the Golgi apparatus. Trends Cell Biol 13, 540-546.

Friebel, A., Ilchmann, H., Aepfelbacher, M., Ehrbar, K., Machleidt, W. \& Hardt, W. D. (2001). SopE and SopE2 from Salmonella typhimurium activate different sets of RhoGTPases of the host cell. J Biol Chem 276, 34035-34040.

Galan, J. E. (2001). Salmonella interactions with host cells: type III secretion at work. Annu Rev Cell Dev Biol 17, 53-86.

Galan, J. E. \& Fu, Y. (2000). Modulation of actin cytoskeleton by Salmonella GTPase activating protein SptP. Methods Enzymol 325, 496-504.

Galan, J. E. \& Zhou, D. (2000). Striking a balance: modulation of the actin cytoskeleton by Salmonella. Proc Natl Acad Sci U S A 97, 8754-8761.

Gallois, A., Klein, J. R., Allen, L. A., Jones, B. D. \& Nauseef, W. M. (2001). Salmonella pathogenicity island 2-encoded type III secretion system mediates exclusion of NADPH oxidase assembly from the phagosomal membrane. J Immunol 166, 5741-5748.

Garcia-del Portillo, F., Zwick, M. B., Leung, K. Y. \& Finlay, B. B. (1993). Salmonella induces the formation of filamentous structures containing lysosomal membrane glycoproteins in epithelial cells. Proc Natl Acad Sci U S A 90, 10544-10548.

Gauthier, A., de Grado, M. \& Finlay, B. B. (2000). Mechanical fractionation reveals structural requirements for enteropathogenic Escherichia coli Tir insertion into host membranes. Infect Immun 68, 4344-4348.

Ghosh, P. (2004). Process of protein transport by the type III secretion system. Microbiol Mol Biol Rev 68, 771-795.

Guignot, J., Caron, E., Beuzon, C., Bucci, C., Kagan, J., Roy, C. \& Holden, D. W. (2004). Microtubule motors control membrane dynamics of Salmonella-containing vacuoles. J Cell Sci 117, 1033-1045.

Hardt, W. D., Chen, L. M., Schuebel, K. E., Bustelo, X. R. \& Galan, J. E. (1998). S. typhimurium encodes an activator of Rho GTPases that induces membrane ruffling and nuclear responses in host cells. Cell 93, 815-826.

Harrington, A. T., Hearn, P. D., Picking, W. L., Barker, J. R., Wessel, A. \& Picking, W. D. (2003). Structural characterization of the N terminus of IpaC from Shigella flexneri. Infect Immun 71, 1255-1264. Harrison, R. E., Brumell, J. H., Khandani, A., Bucci, C., Scott, C. C., Jiang, X., Finlay, B. B. \& Grinstein, S. (2004). Salmonella impairs RILP recruitment to Rab7 during maturation of invasion vacuoles. Mol Biol Cell 15, 3146-3154.

Hensel, M., Shea, J. E., Waterman, S. R. \& other authors (1998). Genes encoding putative effector proteins of the type III secretion system of Salmonella pathogenicity island 2 are required for bacterial virulence and proliferation in macrophages. Mol Microbiol 30, 163-174.

Ho, S. N., Hunt, H. D., Horton, R. M., Pullen, J. K. \& Pease, L. R. (1989). Site-directed mutagenesis by overlap extension using the polymerase chain reaction. Gene 77, 51-59.

Hoiseth, S. K. \& Stocker, B. A. (1981). Aromatic-dependent Salmonella typhimurium are non-virulent and effective as live vaccines. Nature 291, 238-239. 
Hueck, C. J. (1998). Type III protein secretion systems in bacterial pathogens of animals and plants. Microbiol Mol Biol Rev 62, 379-433.

Jiang, X., Rossanese, O. W., Brown, N. F., Kujat-Choy, S., Galan, J. E., Finlay, B. B. \& Brumell, J. H. (2004). The related effector proteins SopD and SopD2 from Salmonella enterica serovar Typhimurium contribute to virulence during systemic infection of mice. Mol Microbiol 54, 1186-1198.

Jones, B. D., Ghori, N. \& Falkow, S. (1994). Salmonella typhimurium initiates murine infection by penetrating and destroying the specialized epithelial M cells of the Peyer's patches. J Exp Med 180, 15-23.

Knodler, L. A. \& Steele-Mortimer, O. (2003). Taking possession: biogenesis of the Salmonella-containing vacuole. Traffic 4, 587-599.

Knodler, L. A. \& Steele-Mortimer, O. (2005). The Salmonella effector PipB2 affects late endosome/lysosome distribution to mediate Sif extension. Mol Biol Cell 16, 4108-4123.

Knodler, L. A., Vallance, B. A., Hensel, M., Jackel, D., Finlay, B. B. \& Steele-Mortimer, O. (2003). Salmonella type III effectors PipB and PipB2 are targeted to detergent-resistant microdomains on internal host cell membranes. Mol Microbiol 49, 685-704.

Kuhle, V. \& Hensel, M. (2002). SseF and SseG are translocated effectors of the type III secretion system of Salmonella pathogenicity island 2 that modulate aggregation of endosomal compartments. Cell Microbiol 4, 813-824.

Kuhle, V., Jackel, D. \& Hensel, M. (2004). Effector proteins encoded by salmonella pathogenicity island 2 interfere with the microtubule cytoskeleton after translocation into host cells. Traffic 5, 356-370.

Lee, S. H. \& Galan, J. E. (2004). Salmonella type III secretionassociated chaperones confer secretion-pathway specificity. Mol Microbiol 51, 483-495.

Linstedt, A. D. \& Hauri, H. P. (1993). Giantin, a novel conserved Golgi membrane protein containing a cytoplasmic domain of at least 350 kDa. Mol Biol Cell 4, 679-693.

Lloyd, S. A., Norman, M., Rosqvist, R. \& Wolf-Watz, H. (2001). Yersinia YopE is targetted for type III secretion by $\mathrm{N}$-terminal, not mRNA, signals. Mol Microbiol 39, 520-531.

Miao, E. A. \& Miller, S. I. (2000). A conserved amino acid sequence directing intracellular type III secretion by Salmonella typhimurium. Proc Natl Acad Sci U S A 97, 7539-7544.

Miao, E. A., Brittnacher, M., Haraga, A., Jeng, R. L., Welch, M. D. \& Miller, S. I. (2003). Salmonella effectors translocated across the vacuolar membrane interact with the actin cytoskeleton. Mol Microbiol 48, 401-415.

Nikolaus, T., Deiwick, J., Rappl, C., Freeman, J. A., Schroder, W., Miller, S. I. \& Hensel, M. (2001). SseBCD proteins are secreted by the type III secretion system of Salmonella pathogenicity island 2 and function as a translocon. J Bacteriol 183, 6036-6045.

Ochman, H., Soncini, F. C., Solomon, F. \& Groisman, E. A. (1996). Identification of a pathogenicity island required for Salmonella survival in host cells. Proc Natl Acad Sci U S A 93, 7800-7804.

Ohlson, M. B., Fluhr, K., Birmingham, C. L., Brumell, J. H. \& Miller, S. I. (2005). SseJ deacylase activity by Salmonella enterica serovar Typhimurium promotes virulence in mice. Infect Immun 73, 62496259.

Ramamurthi, K. S. \& Schneewind, O. (2003). Substrate recognition by the Yersinia type III protein secretion machinery. Mol Microbiol 50, 1095-1102.

Reinicke, A. T., Hutchinson, J. L., Magee, A. I., Mastroeni, P., Trowsdale, J. \& Kelly, A. P. (2005). A Salmonella typhimurium effector protein SifA is modified by host cell prenylation and $S$ acylation machinery. J Biol Chem 280, 14620-14627.
Richter-Dahlfors, A., Buchan, A. M. J. \& Finlay, B. B. (1997). Murine salmonellosis studied by confocal microscopy: Salmonella typhimurium resides intracellularly inside macrophages and exerts a cytotoxic effect on phagocytes in vivo. J Exp Med 186, 569-580.

Ruiz-Albert, J., Yu, X. J., Beuzon, C. R., Blakey, A. N., Galyov, E. E. \& Holden, D. W. (2002). Complementary activities of SseJ and SifA regulate dynamics of the Salmonella typhimurium vacuolar membrane. Mol Microbiol 44, 645-661.

Salcedo, S. P. \& Holden, D. W. (2003). SseG, a virulence protein that targets Salmonella to the Golgi network. EMBO J 22, 5003-5014.

Salcedo, S. P., Noursadeghi, M., Cohen, J. \& Holden, D. W. (2001). Intracellular replication of Salmonella typhimurium strains in specific subsets of splenic macrophages in vivo. Cell Microbiol 3, 587-597.

Seelig, H. P., Schranz, P., Schroter, H., Wiemann, C., Griffiths, G. \& Renz, M. (1994). Molecular genetic analyses of a 376-kilodalton Golgi complex membrane protein (giantin). Mol Cell Biol 14, 2564-2576.

Shea, J. E., Hensel, M., Gleeson, C. \& Holden, D. W. (1996). Identification of a virulence locus encoding a second type III secretion system in Salmonella typhimurium. Proc Natl Acad Sci U S A 93, 2593-2597.

Shea, J. E., Beuzon, C. R., Gleeson, C., Mundy, R. \& Holden, D. W. (1999). Influence of the Salmonella typhimurium pathogenicity island 2 type III secretion system on bacterial growth in the mouse. Infect Immun 67, 213-219.

Sory, M. P., Boland, A., Lambermont, I. \& Cornelis, G. R. (1995). Identification of the YopE and YopH domains required for secretion and internalization into the cytosol of macrophages, using the cyaA gene fusion approach. Proc Natl Acad Sci U S A 92, 11998-12002.

Steele-Mortimer, O., Meresse, S., Gorvel, J.-P., Toh, B.-H. \& Finlay, B. B. (1999). Biogenesis of Salmonella typhimurium-containing vacuoles in epithelial cells involves interactions with the early endocytic pathway. Cell Microbiol 1, 33-49.

Stein, M. A., Leung, K. Y., Zwick, M., Garcia-del Portillo, F. \& Finlay, B. B. (1996). Identification of a Salmonella virulence gene required for formation of filamentous structures containing lysosomal membrane glycoproteins within epithelial cells. Mol Microbiol 20, 151-164.

Stender, S., Friebel, A., Linder, S., Rohde, M., Mirold, S. \& Hardt, W. D. (2000). Identification of SopE2 from Salmonella typhimurium, a conserved guanine nucleotide exchange factor for Cdc42 of the host cell. Mol Microbiol 36, 1206-1221.

Takeuchi, A. (1967). Electron microscope studies of experimental Salmonella infection. I. Penetration into the intestinal epithelium by Salmonella typhimurium. Am J Pathol 50, 109-136.

Tsolis, R. M., Kingsley, R. A., Townsend, S. M., Ficht, T. A., Adams, L. G. \& Baumler, A. J. (1999). Of mice, calves, and men. Comparison of the mouse typhoid model with other Salmonella infections. Adv Exp Med Biol 473, 261-274.

Vasquez-Torres, A., Jones-Carson, J., Baumler, A. J. \& other authors (1999). Extraintestinal dissemination of Salmonella by CD18-expressing phagocytes. Nature 401, 804-808.

Vazquez-Torres, A., Xu, Y., Jones-Carson, J., Holden, D. W., Lucia, S. M., Dinauer, M. C., Mastroeni, P. \& Fang, F. C. (2000). Salmonella pathogenicity island 2-dependent evasion of the phagocyte NADPH oxidase. Science 287, 1655-1658.

Vazquez-Torres, A., Fantuzzi, G., Edwards, C. K., 3rd, Dinarello, C. A. \& Fang, F. C. (2001). Defective localization of the NADPH phagocyte oxidase to Salmonella-containing phagosomes in tumor necrosis factor p55 receptor-deficient macrophages. Proc Natl Acad Sci U S A 98, 2561-2565.

Waterman, S. R. \& Holden, D. W. (2003). Functions and effectors of the Salmonella pathogenicity island 2 type III secretion system. Cell Microbiol 5, 501-511. 